\title{
Phenomenology in supersymmetric neutrinophilic Higgs model with sneutrino dark matter
}

\author{
Ki-Young Choi, ${ }^{a}$ Osamu Seto ${ }^{b}$ and Chang Sub Shin ${ }^{c}$ \\ ${ }^{a}$ Korea Astronomy and Space Science Institute, \\ Daejon 305-348, Republic of Korea \\ ${ }^{b}$ Department of Life Science and Technology, Hokkai-Gakuen University, \\ Sapporo 062-8605, Japan \\ ${ }^{c}$ New High Energy Theory Center, Department of Physics and Astronomy, Rutgers University, \\ Piscataway NJ 08854, U.S.A. \\ E-mail: kiyoungchoi@kasi.re.kr, seto@physics.umn.edu, \\ changsub@physics.rutgers.edu
}

ABSTRACT: We study a supersymmetric neutrinophilic Higgs model with large neutrino Yukawa couplings where neutrinos are Dirac particles and the lightest right-handed $(\mathrm{RH})$ sneutrino is the lightest supersymmetric particle (LSP) as a dark matter candidate. Neutrinophilic Higgs bosons need to be rather heavy by the precise determination of the muon decay width and dark radiation constraints for large Yukawa couplings. From the Large Hadron Collider constraints, neutrinophilic Higgsino mass need to be heavier than several hundred $\mathrm{GeV}$ or close to the RH sneutrino LSP mass. The latter case is interesting because the muon anomalous magnetic dipole moment can be explained with a relatively large lightest neutrino mass, if RH sneutrino mixings are appropriately fine tuned in order to avoid stringent lepton flavor violation constraints. Dark matter is explained by asymmetric $\mathrm{RH}$ sneutrino dark matter in the favoured region by the muon anomalous magnetic dipole moment. In other regions, $\mathrm{RH}$ sneutrino could be an usual WIMP dark matter.

KeYWORDS: Supersymmetry Phenomenology

ARXiv EPRINT: 1406.0228 


\section{Contents}

1 Introduction 2

2 Phenomenological constraints and implications 2

2.1 Neutrino mass and mixing 3

2.2 Muon decay width 4

2.2.1 Loop enhancement of $\mu \rightarrow e \bar{\nu}_{e} \nu_{\mu} \quad 5$

$2.2 .2 \mu \rightarrow \nu_{\alpha} \bar{\nu}_{\beta} e$ with $(\alpha, \beta) \neq(\mu, e) \quad 6$

$2.2 .3 \mu \rightarrow \nu_{R} \bar{\nu}_{R} e \quad 6$

$\begin{array}{lll}2.2 .4 \text { Total } & 7\end{array}$

2.3 Collider constraints 9

$\begin{array}{lll}\text { 2.3.1 Constraints from LEP } & 10\end{array}$

$\begin{array}{lll}2.3 .2 & \text { LHC bound } & 10\end{array}$

2.4 Lepton flavor violation $(\mu \rightarrow e \gamma) \quad 11$

2.5 Anomalous magnetic dipole moment of muon 12

$\begin{array}{ll}2.6 & \text { Compatibility in benchmarks } \\ \end{array}$

3 Cosmological constraints $\quad 15$

$\begin{array}{ll}\text { 3.1 Dark radiation } & 15\end{array}$

$\begin{array}{lll}3.1 .1 & l^{-} l^{+} \longleftrightarrow \nu_{R} \overline{\nu_{R}} & 16\end{array}$

$\begin{array}{lll}3.1 .2 & \nu_{L} \overline{\nu_{L}} \longleftrightarrow \nu_{R} \overline{\nu_{R}} & 17\end{array}$

$\begin{array}{lll}\text { 3.1.3 Decoupling condition } & 17\end{array}$

4 Dark matter $\quad 17$

$\begin{array}{lll}4.1 & \text { Relic density of dark matter } & 17\end{array}$

$\begin{array}{lll}\text { 4.2 Dark matter scattering cross section with a nucleon } & 19\end{array}$

$\begin{array}{ll}4.3 & \text { Indirect signal from sneutrino dark matter } 20\end{array}$

4.4 Decay of cosmic neutrino background 20

$\begin{array}{lll}5 & \text { Conclusion } & 21\end{array}$

A Formula for the muon $g-2 \quad 22$

B Auxiliary functions $\quad 22$ 


\section{Introduction}

A scalar field could be responsible for the breakdown of a large gauge symmetry and the generation of the masses of gauge bosons and fermions. In fact, a scalar boson discovered at the Large Hadron Collider (LHC) appears to be the Higgs boson in the standard model (SM) of particle physics [1, 2]. After the spontaneous symmetry breaking, the mass of each fermion, namely quarks and charged leptons, is given by the product of each Yukawa coupling constant and the vacuum expectation value (VEV) of the Higgs field.

However, one might suspect a special mechanism for the generation of neutrino masses and a special reason for its smallness, because masses of neutrinos are very small compared with other SM fermions. One approach is the so-called seesaw mechanism with very heavy right-handed $(\mathrm{RH})$ Majorana neutrinos, where the smallness of neutrino mass can be understood as a consequence of the high scale of RH neutrino mass [3-5].

Another approach is the neutrinophilic Higgs model [6-8]. In this model, neutrino has Dirac mass terms generated by another Higgs field whose VEV is much smaller than that of the SM Higgs, where the smallness of neutrino mass is a consequence of the smallness of the other Higgs VEV. In this case, the neutrino Yukawa couplings can be much larger than those with only the SM Higgs field if the Higgs VEV is small, because the neutrino mass is the product of the VEV of the neutrinophilic Higgs field and the Yukawa couplings.

The large neutrino Yukawa couplings in the neutrinophilic Higgs model shows many interesting features, such as the possibility of the RH sneutrino as thermal dark matter (DM) [9-11] or the low scale thermal leptogenesis [12-14]. In addition, the large Yukawa couplings have more implications in the flavour structures and astrophysical phenomenon.

In this paper, we examine various phenomenological aspects of the large Yukawa interactions in the supersymmetric extended neutrinophilic Higgs model. Those include the anomalous magnetic moments of muon, lepton flavour violation, experimental constraints on the couplings and the masses of new particles, and cosmological and astrophysical constraints including indirect detection signatures by asymmetric sneutrino DM through gamma ray and neutrinos.

In section 2, we consider the constraints on the Yukawa couplings from neutrino masses and mixings, the muon decay, collider searches, and lepton flavour violation. Taking these constraints into account, we study the possibility to explain the muon anomalous magnetic moment in this model. In section 3, we consider the cosmological constraints from dark radiation, and in section 4 we study the possibility of the lightest $\mathrm{RH}$ sneutrino as DM and the astrophysical constraints on the models. We conclude our study in section 5 . We provide some formulas in the appendices.

\section{Phenomenological constraints and implications}

In a supersymmetric model, the interaction is described by the term in superpotential

$$
W_{N}=\left(\nu_{R}^{c}\right)_{i}\left(y_{\nu}\right)_{i \alpha} L_{\alpha} \cdot H_{\nu}
$$

where $L_{\alpha}$ is the lepton doublet of the Standard Model, $\nu_{R i}$ is a gauge singlet RH neutrino superfield and $H_{\nu}$ is a scalar doublet in addition to the standard two Higgs doublets in 
the MSSM and $i, \alpha=1,2,3$ denotes the generation index. In the so called neutrinophilic Higgs model with the neutrino Dirac mass given by a small VEV of neutrinophilic Higgs field, $\left\langle H_{\nu}^{0}\right\rangle$, neutrino Yukawa couplings can be as large as of the order of unity.

We consider the Yukawa interaction of Dirac neutrino and neutrinophilic Higgs as

$$
\mathcal{L}=\bar{\nu}_{R i}\left(y_{\nu}\right)_{i \alpha} L_{\alpha} H_{\nu}+\text { h.c. }
$$

After the electroweak symmetry breaking, the neutrinophilic Higgs field develops the VEV $\left\langle H_{\nu}^{0}\right\rangle=v_{\nu} / \sqrt{2}$ and generates the neutrino mass. Since neutrinos are Dirac particles in this model, their mass matrix is simply proportional to $3 \times 3$ neutrino Yukawa coupling matrix. The neutrino mass matrix, or equivalently Yukawa interaction, is given by

$$
\mathcal{L}_{\nu \text { mass }}=\bar{\nu}_{R i}\left(m_{\nu}\right)_{i \alpha} \nu_{L \alpha}+\text { h.c. },
$$

with

$$
\left(m_{\nu}\right)_{i \alpha}=\left(y_{\nu}\right)_{i \alpha} \frac{v_{\nu}}{\sqrt{2}},
$$

here and hereafter we assume the Yukawa couplings are real, for simplicity. Therefore, the left- and right-handed neutrinos compose the four component Dirac mass eigenstates.

In a supersymmetric theory, there exists the Yukawa interaction of scalar RH neutrino with the same Yukawa coupling of eq. (2.2), given by

$$
\mathcal{L}=\tilde{\nu}_{R i}\left(y_{\nu}\right)_{i \alpha} \bar{L}_{\alpha} P_{R} \tilde{H}_{\nu}+\text { h.c. } .
$$

Here, RH sneutrinos $\tilde{\nu}_{R i}$ are defined as the superpartner of each $\nu_{R i}$. If the lightest $\mathrm{RH}$ sneutrino is the lightest supersymmetric particle (LSP), it can be a good candidate for DM as shown in refs. $[9,10]$. Throughout this paper, we consider this case of RH sneutrino DM and study the phenomenological constraints and implications.

\subsection{Neutrino mass and mixing}

Without loss of the generality, we can regard that the $\nu_{R i}$ is already mass eigenstate. The neutrino mass matrix is diagonalized with the Maki-Nakagawa-Sakata (MNS) matrix $U^{\mathrm{MNS}}$, which transfers LH neutrinos from mass eigenstates $\left(\nu_{L, i}\right)$ to flavor eigenstates $\left(\nu_{L, \alpha}\right)$ $\nu_{L, \alpha}=\left(U^{\mathrm{MNS}}\right)_{\alpha i} \nu_{L, i}$

$$
\operatorname{diag}\left(m_{1}, m_{2}, m_{3}\right)_{i j}=\left(m_{\nu}\right)_{i \alpha}\left(U^{\mathrm{MNS}}\right)_{\alpha j} .
$$

The neutrino oscillation data gives two independent mass squared differences and three mixing angles [15],

$$
\begin{aligned}
m_{2}^{2}-m_{1}^{2} & \simeq 7.5 \times 10^{-5} \mathrm{eV}^{2}, \\
\left|m_{3}^{2}-m_{1}^{2}\right| & \simeq 2.3 \times 10^{-3} \mathrm{eV}^{2}, \\
\sin ^{2} 2 \theta_{23} & >0.95 \\
\sin ^{2} 2 \theta_{12} & \simeq 0.857 \\
\sin ^{2} 2 \theta_{13} & \simeq 0.095
\end{aligned}
$$




\begin{tabular}{|c|c|c|c|c|}
\hline Point & 1: normal & 2: normal & 3: inverted & 4: inverted \\
\hline$m_{1,3}[\mathrm{eV}]$ & $m_{1}=0.0$ & $m_{1}=0.07$ & $m_{3}=0.05$ & $m_{3}=0.0$ \\
\hline \multirow{3}{*}{$\left(y_{\nu}\right)_{i \alpha}$} & $\left(\begin{array}{ccc}0.0 & 0.0 & 0.0 \\
0.14 & 0.13 & -0.16 \\
0.22 & 1.0 & 1.0\end{array}\right)$ & $\left(\begin{array}{ccc}0.96 & -0.57 & 0.36 \\
0.65 & 0.62 & -0.77 \\
0.22 & 1.0 & 1.0\end{array}\right)$ & $\left(\begin{array}{ccc}1.0 & -0.59 & 0.37 \\
0.68 & 0.65 & -0.80 \\
0.14 & 0.62 & 0.62\end{array}\right)$ & $\left(\begin{array}{ccc}1.0 & -0.59 & 0.37 \\
0.68 & 0.65 & -0.80 \\
0.0 & 0.0 & 0.0\end{array}\right)$ \\
\hline$\sum m_{\nu}[\mathrm{eV}]$ & 0.06 & 0.23 & 0.19 & 0.10 \\
\hline$v_{\nu}[\mathrm{eV}]$ & 0.05 & 0.08 & 0.08 & 0.05 \\
\hline
\end{tabular}

Table 1. Neutrino Yukawa coupling matrix of the four benchmark 1, 2, 3 and 4 used in this work are shown. The resultant $\sum m_{\nu}$ for a given input parameter $m_{1}$ or $m_{3}$ is also listed. $v_{\nu}$ is a free parameter. The noted values of $v_{\nu}$ are examples in the case that we normalize the largest element of Yukawa matrix to be 1.0 .

The neutrino Yukawa couplings can be expressed in terms of these neutrino oscillation parameters as

$$
\begin{aligned}
\left(y_{\nu}\right)_{i \alpha} & =\frac{\sqrt{2}}{v_{\nu}} \operatorname{diag}\left(m_{1}, m_{2}, m_{3}\right)_{i j}\left(U^{\mathrm{MNS}}\right)_{j \alpha}^{-1} \\
& \simeq \frac{1}{v_{\nu}}\left(\begin{array}{ccc}
\sqrt{2} m_{1} \cos \theta_{12} & -m_{1} \sin \theta_{12} & m_{1} \sin \theta_{12} \\
\sqrt{2} m_{2} \sin \theta_{12} & m_{2} \cos \theta_{12} & -m_{2} \cos \theta_{12} \\
\sqrt{2} m_{3} \sin \theta_{13} & m_{3} & m_{3}
\end{array}\right) .
\end{aligned}
$$

Here, we neglect for simplicity any $\mathrm{CP}$ phase and take $y_{\nu}$ to be real. In the second line, $\theta_{23} \simeq \pi / 4$ and $\sin \theta_{13} \ll 1$ are used, while we use the full formula of MNS matrix in our numerical calculation.

The upper bound on the sum of neutrino masses is provided by cosmological arguments. Since its value strongly depends on data set and a cosmological model used in the analysis, for reference, we here just quote one of less conservative values

$$
\sum m_{\nu}<0.23 \mathrm{eV}
$$

from ref. [16].

In table 1, we list four benchmark points used in our analysis for a given lightest neutrino mass, $m_{1}=0,0.07 \mathrm{eV}$ for normal hierarchy $\left(m_{1}<m_{2}<m_{3}\right)$ and $m_{3}=0,0.05 \mathrm{eV}$ for inverted hierarchy $\left(m_{3}<m_{1}<m_{2}\right)$ of neutrino masses. To estimate Yukawa coupling constants, we have to fix one extra free parameter $v_{\nu}$. Example value sets of $y_{\nu}$ in table 1 are obtained under the assumption that the largest coupling is unity. The actual value can be somewhat larger or smaller by changing $v_{\nu}$.

\subsection{Muon decay width}

Muon decays into electron, electron-type anti-neutrino and muon-type neutrino, $\mu \rightarrow e \bar{\nu}_{e} \nu_{\mu}$ in the Standard Model. However, in the neutrinophilic Higgs model, the Yukawa interaction eq. (2.1) gives additional contribution to the SM prediction via one loop induced vertices and changes the muon decay width. Those come from box diagrams with a loop $\left(\nu_{R}, H_{\nu}^{0}\right.$ and $\left.H_{\nu}^{-}\right)$or $\left(\tilde{\nu}_{R}, \tilde{H}_{\nu}^{0}\right.$ and $\left.\tilde{H}_{\nu}^{-}\right)$. Moreover, muon has additional decay mode into electron, 
$\mathrm{RH}$ neutrino and anti-RH neutrino, through the $H_{\nu}^{+}$mediation. This new contribution can be severely constrained by the precise measurement of the decay rate and inverse decay rate of muon.

Now we are going to estimate those additional contributions to muon decay width. For this, we define mass eigenstates $\tilde{N}_{i}$ from flavour states $\nu_{R i}$ with an unitary matrix $S_{i j}$ as defined by

$$
\begin{aligned}
\tilde{\nu}_{R i} & =S_{i j} \tilde{N}_{j}, \\
S_{i j} & =R\left(\sigma_{12}\right) R\left(\sigma_{23}\right) R\left(\sigma_{13}\right),
\end{aligned}
$$

with $R$ being a rotation matrix and the variables, $\sigma_{12}, \sigma_{23}, \sigma_{13}$, are the corresponding mixing angle. The Yukawa interaction eq. (2.5) becomes

$$
\mathcal{L}=\tilde{N}_{k}\left(S^{T}\right)_{k i}\left(y_{\nu}\right)_{i \alpha} \bar{L}_{\alpha} P_{R} \tilde{H}_{\nu}+\text { h.c. }
$$

for the mass eigenstates. Thus, we define $\tilde{y}_{i \alpha} \equiv\left(S^{T} y_{\nu}\right)_{i \alpha}$ for Yukawa couplings of the $\mathrm{RH}$ sneutrino, lepton and Higgsino.

\subsubsection{Loop enhancement of $\mu \rightarrow e \bar{\nu}_{e} \nu_{\mu}$}

First, let us estimate the decay width of the main decay mode. In the following, $p_{1}, p_{2}, q_{1}$ and $q_{2}$ are the momentum of incoming $\mu$, outgoing $e, \nu_{\mu}$ and $\bar{\nu}_{e}$ respectively. The amplitude of $W$ boson mediated process is given by

$$
i \mathcal{M}_{1}=\bar{u}\left(q_{1}\right) \frac{g_{2}}{\sqrt{2}} \gamma^{\mu} P_{L} u\left(p_{1}\right) \frac{i g_{\mu \nu}}{M_{W}^{2}} \bar{u}\left(p_{2}\right) \frac{g_{2}}{\sqrt{2}} \gamma^{\nu} P_{L} v\left(q_{2}\right)
$$

where $g_{2}$ is the $\mathrm{SU}(2)$ gauge coupling and $M_{W}$ is the $W$ boson mass. That of $\mathrm{RH}$ neutrino and Higgs bosons loop shown in the left window of figure 1 is given by

$$
\begin{aligned}
i \mathcal{M}_{2} \simeq & \sum_{i, j} \bar{u}\left(q_{1}\right) \frac{\left(y_{\nu}\right)_{i \mu}}{\sqrt{2}} \gamma_{\mu}\left(y_{\nu}\right)_{i \mu} P_{L} u\left(p_{1}\right) \bar{u}\left(p_{2}\right)\left(y_{\nu}\right)_{j e} \gamma_{\nu} \frac{\left(y_{\nu}\right)_{j e}}{\sqrt{2}} P_{L} v\left(q_{2}\right) \\
& \times \frac{-i}{4(4 \pi)^{2}}\left(F_{2}\left(M_{H_{\nu}^{-}}, M_{H_{\nu}^{0}}\right)+F_{2}\left(M_{H_{\nu}^{-}}, M_{A_{\nu}^{0}}\right)\right) g_{\mu \nu}
\end{aligned}
$$

with the auxiliary function $F_{2}(x)$, which is defined in the appendix. In this estimation of $F_{2}(x)$, we neglect $\mathcal{O}\left(M^{-4}\right)$ terms with $M$ being the mass scale of new particles, those are much smaller than the leading corrections of $\mathcal{O}\left(M^{-2}\right)$. That of $\mathrm{RH}$ sneutrino and Higgsino loop shown in the right window of figure 1 is given by

$$
\begin{aligned}
i \mathcal{M}_{3} \simeq & \bar{u}\left(p_{2}\right) \gamma_{\mu} P_{L} u\left(p_{1}\right) \bar{u}\left(q_{1}\right) \gamma_{\nu} P_{L} v\left(q_{2}\right) g_{\mu \nu} \\
& \times \frac{-i \sum_{i, j}\left(S^{T} y_{\nu}\right)_{i \mu}^{2}\left(S^{T} y_{\nu}\right)_{j e}^{2} F_{3}\left(M_{\tilde{H}_{\nu}^{-}}, M_{\tilde{H}_{\nu}^{0}}, M_{\tilde{N}_{i}}, M_{\tilde{N}_{j}}\right)}{4(4 \pi)^{2}} .
\end{aligned}
$$



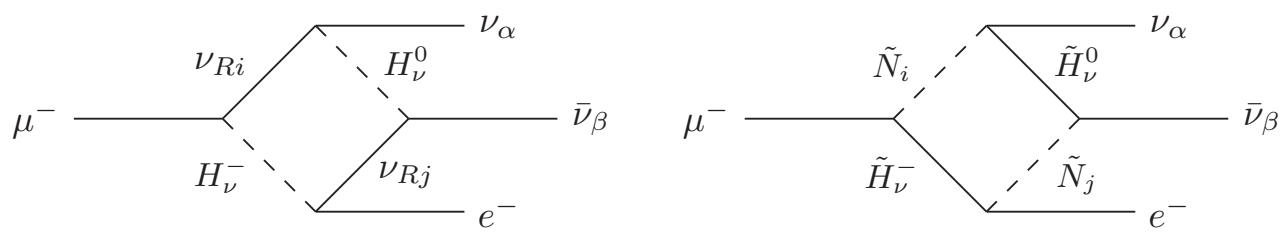

Figure 1. The Feynman diagrams of muon decay to electron and left-handed neutrinos via one loop.

We obtain

$$
\begin{aligned}
\Gamma\left(\mu \rightarrow \nu_{\mu} \bar{\nu}_{e} e\right) \simeq \Gamma_{\mu}^{(\mathrm{SM})}[ & 1-2 \frac{2 M_{W}^{2}}{g_{2}^{2}} \frac{\sum_{i, j}\left(y_{\nu}\right)_{i \mu}^{2}\left(y_{\nu}\right)_{j e}^{2}}{8(4 \pi)^{2}}\left(F_{2}\left(M_{H_{\nu}^{-}}, M_{H_{\nu}^{0}}\right)+F_{2}\left(M_{H_{\nu}^{-}}, M_{A_{\nu}^{0}}\right)\right) \\
& \left.+\frac{1}{2} \frac{2 M_{W}^{2}}{g_{2}^{2}} \frac{\sum_{i, j}\left(S^{T} y_{\nu}\right)_{i \mu}^{2}\left(S^{T} y_{\nu}\right)_{j e}^{2} F_{3}\left(M_{\tilde{H}_{\nu}^{-}}, M_{\tilde{H}_{\nu}^{0}}, M_{\tilde{N}_{i}}, M_{\tilde{N}_{j}}\right)}{4(4 \pi)^{2}}\right] \\
\Gamma_{\mu}^{(\mathrm{SM})}= & \frac{m_{\mu}^{5}}{192 \pi^{3}} \frac{1}{2 v^{4}},
\end{aligned}
$$

with the auxiliary function $F_{3}(x, y, z, w)$, which is given in the appendix. Here $m_{\mu}$ is the muon mass and $v \simeq 246 \mathrm{GeV}$ is the VEV of the SM Higgs field and we keep only the leading order loop corrections, namely the interference between tree and one loop.

\subsection{2 $\mu \rightarrow \nu_{\alpha} \bar{\nu}_{\beta} e$ with $(\alpha, \beta) \neq(\mu, e)$}

Due to the lepton flavor violating neutrino Yukawa coupling, the flavor of the final state neutrino can be different from muon-type and anti-electron-type. However, this decay mode has only loop induced new contribution and is suppressed compared to the other contribution to the decay which has the interference term between tree-level and loop induced term. Thus, this mode is negligible.

\subsection{3 $\mu \rightarrow \nu_{R} \bar{\nu}_{R} e$}

This decay mode with $\mathrm{RH}$ neutrinos in the final state is induced by the tree level process mediated by the neutrinophilic charged Higgs boson [17]. A worth noting feature is that this is not a $V-A$ interaction but a scalar interaction. The amplitude of the process $\mu \rightarrow \nu_{R i} \bar{\nu}_{R j}$ e mediated by $H_{\nu}^{-}$shown in the figure 2 is given by

$$
\begin{aligned}
i \mathcal{M}_{H_{\nu}} & =\bar{u}\left(q_{1}\right)\left(y_{\nu}\right)_{\mu i} P_{L} u\left(p_{1}\right) \frac{i}{M_{H_{\nu}^{+}}^{2}} \bar{u}\left(p_{2}\right)\left(y_{\nu}\right)_{e j} P_{R} v\left(q_{2}\right) \\
& \equiv \frac{4 G_{F}}{\sqrt{2}} g_{L L}^{S} \bar{u}\left(q_{1}\right) P_{L} u\left(p_{1}\right) \bar{u}\left(p_{2}\right) P_{R} v\left(q_{2}\right) .
\end{aligned}
$$

Here, we normalise the effective coupling with $G_{F}$, the Fermi constant measured in the experiment, and we introduce a new parameter $g_{L L}^{S}$ defined by [18]

$$
g_{L L}^{S} \equiv \frac{\left(y_{\nu}\right)_{i \mu}\left(y_{\nu}\right)_{j e}}{M_{H_{\nu}^{+}}^{2}} \frac{\sqrt{2}}{4 G_{F}} .
$$




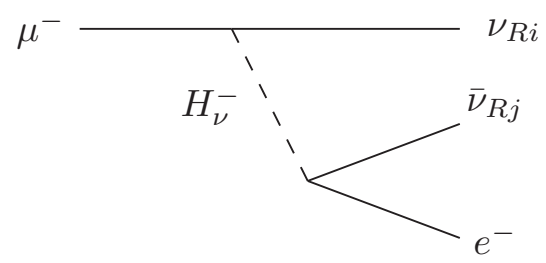

Figure 2. The Feynman diagrams of muon decay to electron and right-handed neutrinos.

The partial width is estimated as

$$
\Gamma\left(\mu \rightarrow \nu_{R i} \bar{\nu}_{R j} e\right)=\frac{\sum_{i, j}\left|\left(y_{\nu}\right)_{\mu i}\left(y_{\nu}\right)_{e j}\right|^{2}}{64 M_{H_{\nu}^{+}}^{4}} \frac{m_{\mu}^{5}}{192 \pi^{2}} .
$$

As we will see later, it turns out that this decay mode has to be highly suppressed due to the well consistency with the SM. Thus, in fact, this would not be significant for muon decay contribution.

\subsubsection{Total}

From eqs. (2.17) and (2.21), the final total decay width of muon is given by

$$
\begin{aligned}
& \frac{\Gamma_{\mu}}{\Gamma_{\mu}^{(\mathrm{SM})}} \simeq\left[1-\frac{\sum_{i, j}\left(y_{\nu}\right)_{\mu i}^{2}\left(y_{\nu}\right)_{e j}^{2}}{8(4 \pi)^{2}} v^{2}\left(F_{2}\left(M_{H_{\nu}^{-}}, M_{H_{\nu}^{0}}\right)+F_{2}\left(M_{H_{\nu}^{-}}, M_{A_{\nu}^{0}}\right)\right)\right. \\
& +\frac{v^{2} \sum_{i, j}\left(S^{T} y_{\nu}\right)_{i \mu}^{2}\left(S^{T} y_{\nu}\right)_{j e}^{2} F_{3}\left(M_{\tilde{H}_{\nu}^{-}}, M_{\tilde{H}_{\nu}^{0}}, M_{\tilde{N}_{i}}, M_{\tilde{N}_{j}}\right)}{16(4 \pi)^{2}} \\
& \left.+\frac{v^{4} \sum_{i, j}\left(y_{\nu}\right)_{\mu i}^{2}\left(y_{\nu}\right)_{e j}^{2}}{32 M_{H_{\nu}^{+}}^{4}}\right] .
\end{aligned}
$$

By comparing Fermi constant $G_{F}$ measured from muon decay width and other SM quantities, the consistency of the SM can be tested [19, 20]. If we express the Fermi coupling constant with a parameter which stands for a correction due to new physics by

$$
G_{F}=\frac{g_{2}^{2}}{4 \sqrt{2} M_{W}^{2}(1-\Delta)},
$$

the new physics contribution $\Delta$ is constrained to be [21]

$$
\Delta=0 \pm 0.0006 .
$$

The last term in eq. (2.22) comes from the non- $(V-A)$ interaction via $H_{\nu}^{+}$mediation. The muon decay experiments can not measure helicity of produced neutrinos, but the inverse decay of muon, $\nu_{\mu}+e \rightarrow \mu+$ missing, well confirms the $V-A$ form interaction and leave a small room for scalar interaction as [22]

$$
\left|g_{\mathrm{LL}}^{S}\right|^{2}<0.475 \quad \text { (90\% C.L.) } \text {. }
$$

With eq. (2.20), we find the constraint on the $H_{\nu}^{+}$mass and Yukawa couplings. 


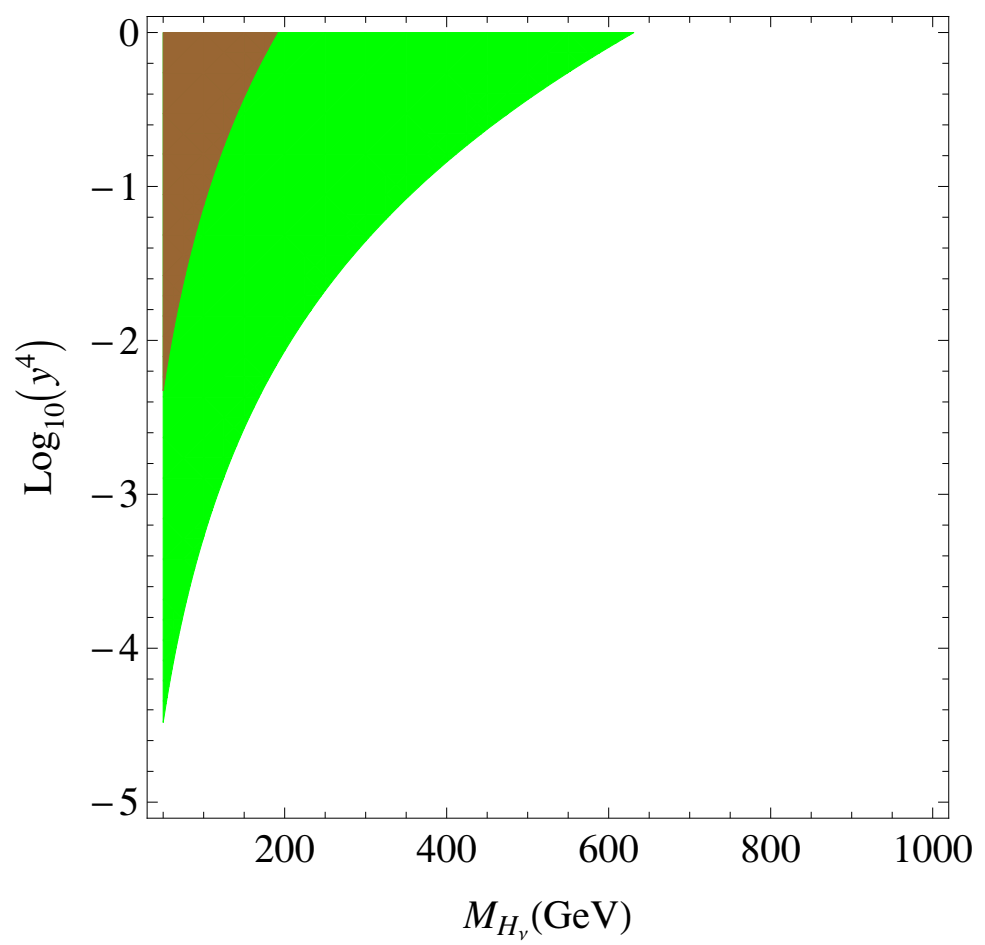

Figure 3. Constraints on charged Higgs boson mass from the muon decay property. The brown (green) shaded region is excluded by too large $g_{L L}^{S}(\Delta)$.

First, let us consider the constraints on charged Higgs boson from muon decay in the decoupling limit of Higgsino of the second term. Then the first term in eq. (2.22) gives dominant contribution to $\Delta$ and the third term to $g_{L L}^{S}$. The constraints on charged Higgs mass and Yukawa coupling is shown in figure 3, where for simplicity we take $M_{\tilde{H}_{\nu}^{-}}=M_{\tilde{H}_{\nu}^{0}}$. For Yukawa couplings of the order of unity, the mass of charged Higgs must be heavier than around $600 \mathrm{GeV} .{ }^{1}$ The cosmological consideration of dark radiation imposes the further stringent lower bound on the masses of those extra Higgs bosons, as we will see later in section 3 .

Next, we need to consider the decoupling limit of very heavy Higgs boson as found just above, the the dominant correction comes from the second term in eq. (2.22) from the sneutrino and chargino loop, namely

$$
2 \Delta \simeq \frac{v^{2} F_{3}\left(M_{\tilde{H}_{\nu}^{-}}, M_{\tilde{H}_{\nu}^{0}}, M_{\tilde{N}_{i}}, M_{\tilde{N}_{j}}\right)}{16(4 \pi)^{2}} .
$$

In figure 4 , we show the contour plot of $2 \Delta$ for $\mathcal{O}(1)$ Yukawa couplings in the plane of $\left(M_{\tilde{H}_{\nu}^{-}}\right.$, $\left.M_{\tilde{N}_{i}}\right)$ with $M_{\tilde{N}_{i}}=M_{\tilde{N}_{j}}$ in the left window, and that in the plane of $\left(M_{\tilde{N}_{i}} / M_{\tilde{H}_{\nu}^{-}}, M_{\tilde{N}_{j}} / M_{\tilde{H}_{\nu}^{-}}\right)$ with $M_{\tilde{H}_{\nu}^{-}}=100 \mathrm{GeV}$ in the right window. We can see that the charged Higgsino $\tilde{H}_{\nu}^{-}$need to be heavier than a few hundreds $\mathrm{GeV}$ for degenerate sneutrino case, $M_{\tilde{N}_{j}} \simeq M_{\tilde{N}_{i}}$, or two of RH sneutrinos need to be several times heavier than chargino with $100 \mathrm{GeV}$ mass and only one RH neutrino can be light.

\footnotetext{
${ }^{1}$ Neutrinophilic Higgs bosons with mass $\mathcal{O}(100) \mathrm{GeV}$ is possible for $y_{\nu}<\mathcal{O}(0.01)$ [23-25].
} 

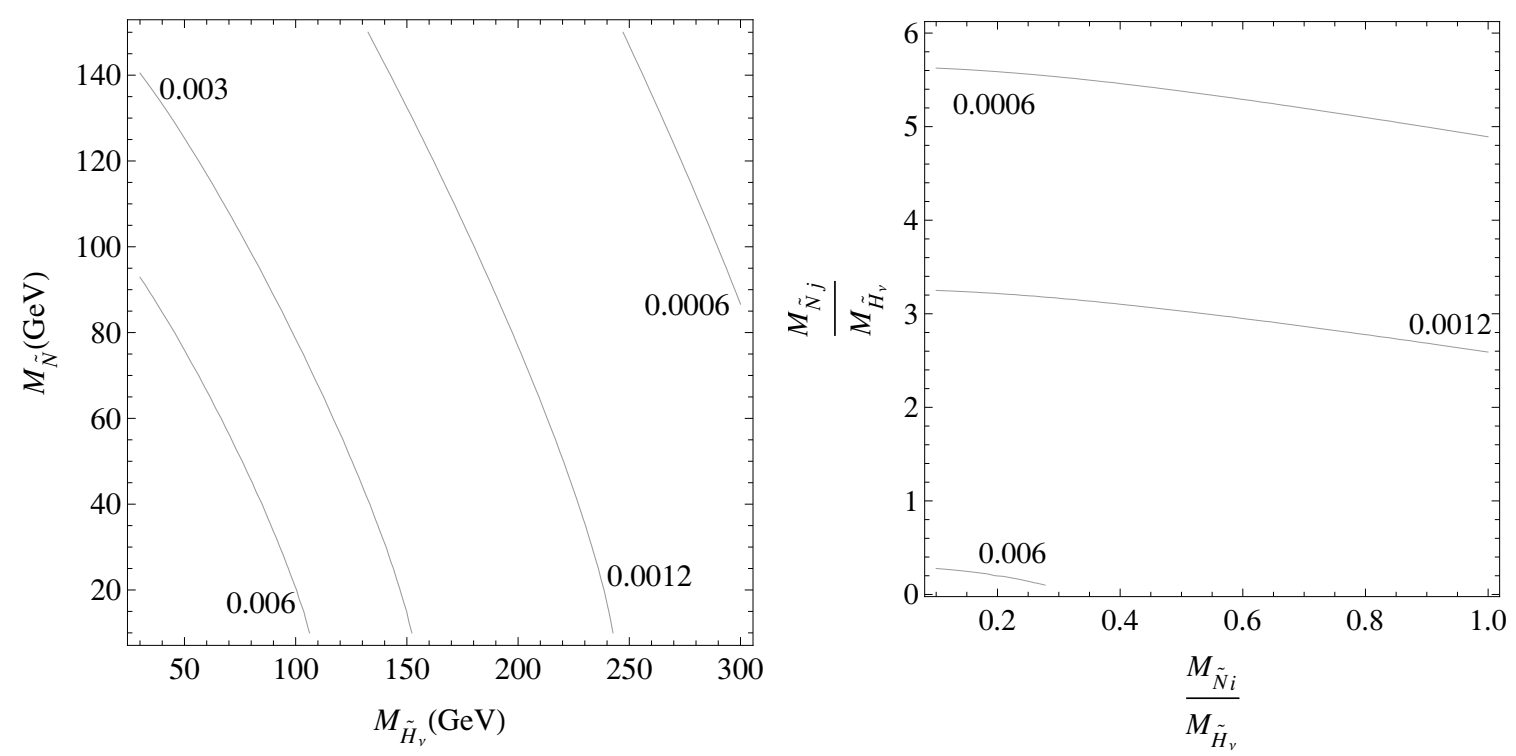

Figure 4. Contours of $2 \Delta$ of eq. (2.26) in the plane of $\left(M_{\tilde{H}_{\nu}^{-}}, M_{\tilde{N}_{i}}\right)$ with $M_{\tilde{\nu}_{i}}=M_{\tilde{N}_{j}}$ in the left window, and in the plane of $\left(M_{\tilde{N}_{j}} / M_{\tilde{H}_{\nu}^{-}}, M_{\tilde{N}_{j}} / M_{\tilde{H}_{\nu}^{-}}\right)$with $M_{\tilde{H}_{\nu}^{-}}=100 \mathrm{GeV}$ in the right window. The region $2 \Delta \lesssim 0.0012$ is preferred.

\subsection{Collider constraints}

In our model, the neutrinophilic Higgsinos $\left(\tilde{H}_{\nu}^{0}, \tilde{H}_{\nu}^{+}\right)$are $\mathrm{SU}(2)$ doublet and can be light enough to be produced at the on-shell from $p p$ or $e^{+} e^{-}$collisions, and subsequently decay to leptons $\left(l_{i}\right)$ and lightest sneutrino $\left(\tilde{N}_{\mathrm{DM}}\right)$, which similar to the production of Wino/Zino and subsequent decay to the lightest neutralino in the minimal supersymmetric standard model (MSSM). However, the collider constraints on our model with the RH sneutrino LSP is slightly different from the current searches on SUSY based on the neutralino LSP.

The production channels of the neutrinophilic Higgsinos are $s$-channel $Z^{0} / \gamma$ boson exchange for $e^{+} e^{-}, p p$ collisions, $t$-channel $\tilde{N}$ exchange for $e^{+} e^{-}$collision, and $s$-channel $W^{ \pm}$exchange for $p p$ collision. They subsequently decay to leptons $\left(l_{i}\right)$ and the lightest sneutrino $\left(\tilde{N}_{\mathrm{DM}}\right)$,

$$
\begin{aligned}
& \tilde{H}_{\nu}^{+} \rightarrow \tilde{N}_{i} l_{i}^{+}, \quad \tilde{H}_{\nu}^{0} \rightarrow \tilde{N}_{i} \bar{\nu}_{j}, \\
& \tilde{N}_{i}^{*} \rightarrow l_{i}^{+} \overline{\tilde{H}}_{\nu}^{+}\left(\bar{\nu}_{j} \tilde{\tilde{H}}_{\nu}^{0}\right) \quad \text { or } \quad l_{i}^{+} e^{-} \tilde{N}_{\mathrm{DM}}^{*}\left(\bar{\nu}_{j} \nu_{k} \tilde{N}_{\mathrm{DM}}^{*}\right) \quad \text { for } i \neq \mathrm{DM},
\end{aligned}
$$

where $\nu_{i}$ is the mass eigenstate of neutrino, and we assume that $\tilde{N}_{\text {DM }}$ is the lightest RH sneutrino as dark matter. For a case where those particle are too heavy to be produced at the on-shell, the production cross section is kinematically very suppressed. For cases that $\mathrm{RH}$ sneutrinos are light but degenerate, only three-body decay is possible via virtual Higgsino $\left(\tilde{H}_{\nu}\right)$ and the energy of the produced lepton is much suppressed.

The corresponding diagrams are given in figure 5 . The final decay products are multi leptons plus a large missing energy by $\tilde{N}_{\mathrm{DM}}$,

$$
\begin{gathered}
e^{+} e^{-}(q \bar{q}) \rightarrow l_{i}^{+} l_{j}^{-}+\text {missing } \mathrm{E}+\left(n l^{+} l^{-}\right), \\
u \bar{d} \rightarrow l_{i}^{+}+\text {missing } \mathrm{E}+\left(n l^{+} l^{-}\right),
\end{gathered}
$$



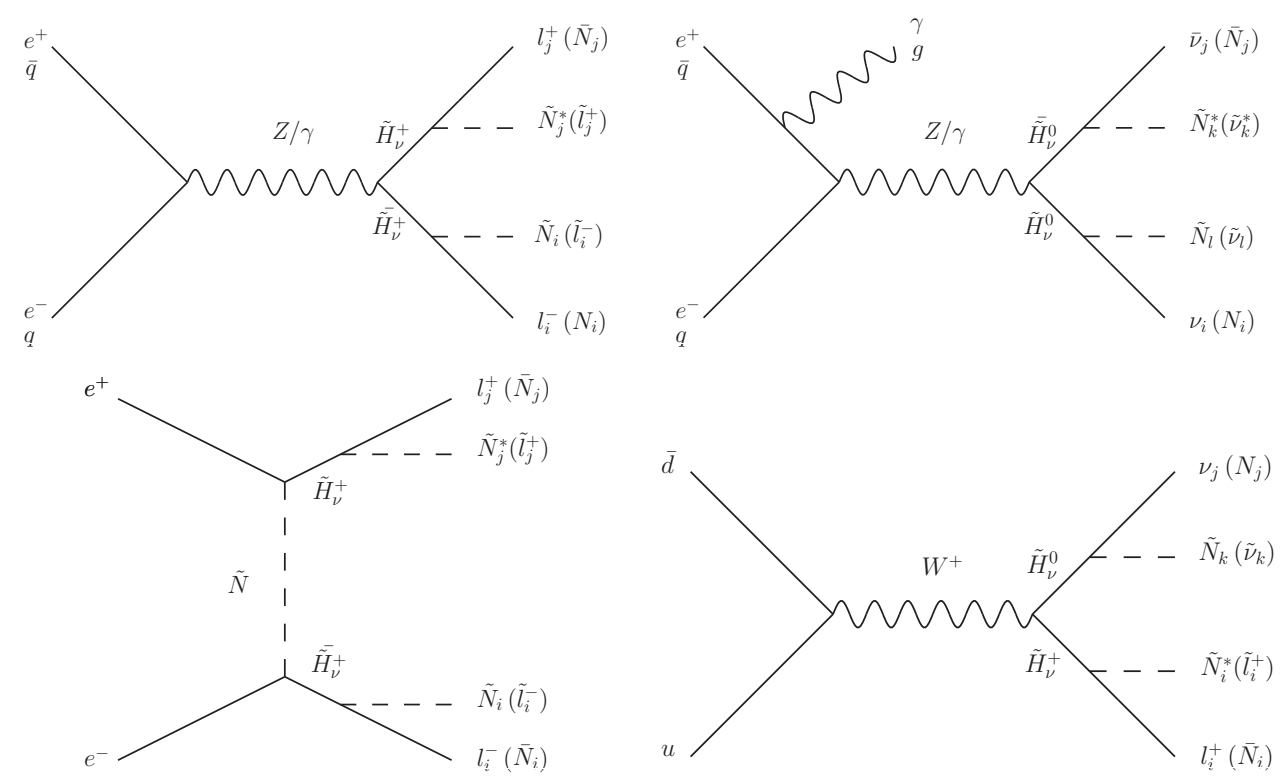

Figure 5. The neutrinophilic Higgsinos $\left(\tilde{H}_{\nu}\right)$ production and its sequent decay into RH sneutrino. $\tilde{H}_{\nu}$ s are produced by the $s$-channel of $Z^{0} / \gamma, t$-channel of $\tilde{N}$, and $s$-channel of $W^{ \pm}$exchanges.

with $n$ being an integer, or mono photon plus a large missing energy by $\tilde{N}_{\text {DM }}$,

$$
e^{+} e^{-}(q \bar{q}) \rightarrow \gamma+\text { missing E. }
$$

\subsubsection{Constraints from LEP}

As far as the LEP bound on chargino is concerned, if the mass of the Higgsinos are greater than the threshold energy scale for $e^{+} e^{-}$collision $(\sqrt{s} / 2 \simeq 104 \mathrm{GeV})$, it is natural to expect that the constraints are relaxed drastically. Thus, we take $m_{\tilde{H}_{\nu}} \gtrsim 103.5 \mathrm{GeV}[26]$ as the kinematical lower bound to avoid the LEP searches for direct production of charginos.

For the direct production of $\tilde{N}_{i}$, the $t$-channel $\tilde{H}_{\nu}$ exchange for $e^{+} e^{-}$collision is dominant as in figure 6.

$$
e^{+} e^{-} \rightarrow \gamma+\text { missing E. }
$$

In this case, the result of monophoton searches for the MSSM neutralino $e^{+} e^{-} \rightarrow \tilde{\chi}_{1}^{0} \tilde{\chi}_{1}^{0} \gamma$ can be used to constrain the mass and couplings of the RH sneutrino to electrons [27]. In this analysis, using the effective operator $\bar{\chi} e \bar{e} \chi / \Lambda$, the cutoff scale $\Lambda$ should be greater than about $330 \mathrm{GeV}$ for the fermion dark matter mass $m_{\chi}<80 \mathrm{GeV}$ as shown in figure 7 .

\subsubsection{LHC bound}

The current $8 \mathrm{TeV}$ LHC gives lower bound on the charged Higgsino mass of $550 \mathrm{GeV}$, leaving the degenerate region with $M_{\tilde{H}}-M_{\tilde{N}}<50 \mathrm{GeV}$ unconstrained from the chargino decay into light leptons, namely $e$ or $\mu$. For the decay into $\tau$ lepton, the bound is relaxed and requires $M_{\tilde{H}} \gtrsim 350 \mathrm{GeV}$ and $M_{\tilde{N}} \gtrsim 150 \mathrm{GeV}[28]$. 

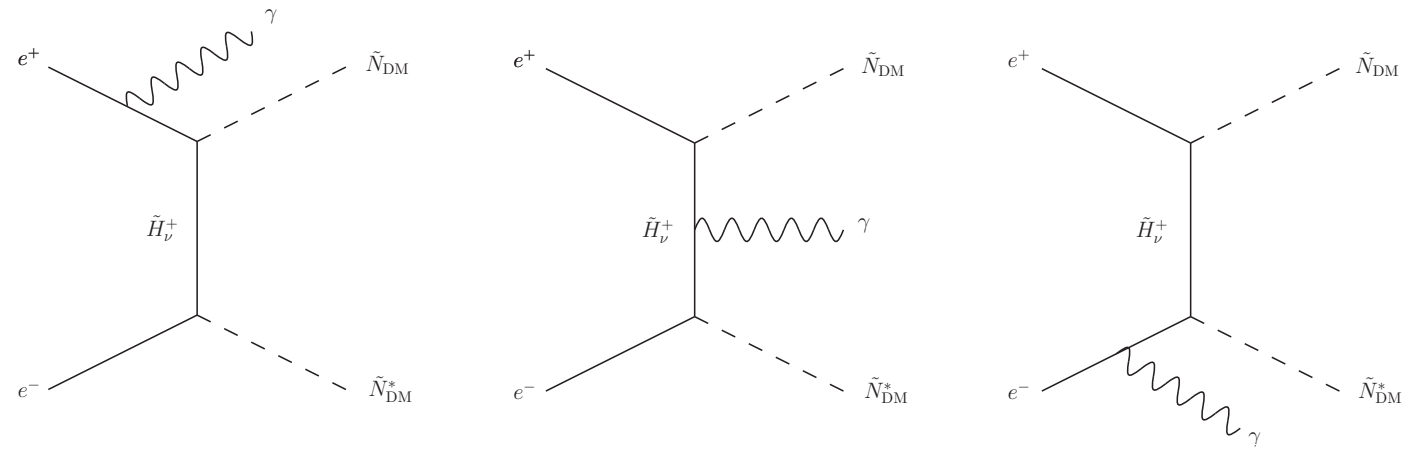

Figure 6. Feynman diagrams for $e^{+} e^{-} \rightarrow \tilde{N}_{\mathrm{DM}} \tilde{N}_{\mathrm{DM}} \gamma$. Since the produced RH sneutrinos are the lightest superpartners, $\tilde{N}_{\mathrm{DM}}$, the only observable signal is photon.

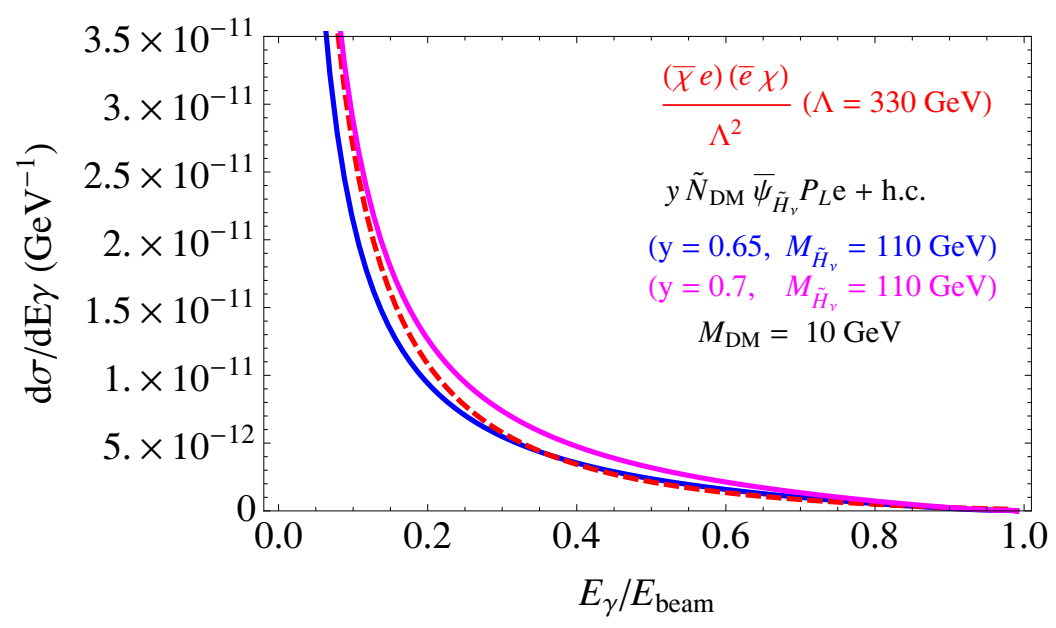

Figure 7. Production rate $\left(d \sigma / d E_{\gamma}\right)$ with respect to $E_{\gamma} / E_{\text {beam }}$ for $\left(e^{+} e^{-} \rightarrow \gamma\right.$ plus DM pair $)$ in two models. Red dashed line corresponds to the model studied in ref. [27]. The studied process is $\left(e^{+} e^{-} \rightarrow \gamma \chi \bar{\chi}\right)$ from the interaction term $(\bar{\chi} e)(\bar{e} \chi) / \Lambda^{2}$. Depending on the dark matter mass $M_{\mathrm{DM}}$, the the lower limit of $\Lambda$ is different. The DELPHI(LEP) monophoton search for $M_{\mathrm{DM}}=10 \mathrm{GeV}$ gives the lower bound on $\Lambda \geq 330 \mathrm{GeV}$. The second model is ours. The event is $\left(e^{+} e^{-} \rightarrow \gamma \tilde{N}_{e} \tilde{N}_{e}^{*}\right)$ from the interaction term $y \tilde{N}_{\mathrm{DM}} \bar{\psi}_{\tilde{H}_{\nu}} P_{L} e+$ h.c.. We take $m_{\tilde{H}_{\nu}}=110 \mathrm{GeV}$, and the cases with two Yukawa couplings $(y=0.65,0.7)$ are presented (blue and magenta). $E_{\text {beam }}$ is taken as $100 \mathrm{GeV}$, which is the average value for the LEP search. We find that our model parameters $\left(y\right.$ and $m_{\tilde{H}_{\nu}}$ ) are constrained by the LEP search as did in ref. [27], i.e. the region above the red dashed line is ruled out.

\subsection{Lepton flavor violation $(\mu \rightarrow e \gamma)$}

The off-diagonal components of Yukawa couplings $\left(y_{\nu}\right)_{i \alpha}$ in eq. (2.2) induce lepton flavor violating (LFV) decay of leptons. The general effective operator can be written as

$$
\mathcal{M}=e \epsilon^{\mu *} \overline{u_{i}}(p-q)\left[i m_{j} \sigma_{\mu \nu} q^{\nu}\left(A_{2}\right)_{i j}+i m_{j} \sigma_{\mu \nu} q^{\nu} \gamma_{5}\left(A_{3}\right)_{i j}\right] u_{j}(p),
$$

with $\sigma_{\mu \nu}=\frac{i}{2}\left[\gamma_{\mu}, \gamma_{\nu}\right]$. The decay rate of $l_{j} \rightarrow l_{i} \gamma$ is given by

$$
\Gamma\left(l_{j} \rightarrow l_{i} \gamma\right)=\frac{e^{2}}{8 \pi} m_{l_{j}}^{5}\left(\left|\left(A_{2}\right)_{i j}\right|^{2}+\left|\left(A_{3}\right)_{i j}\right|^{2}\right) .
$$



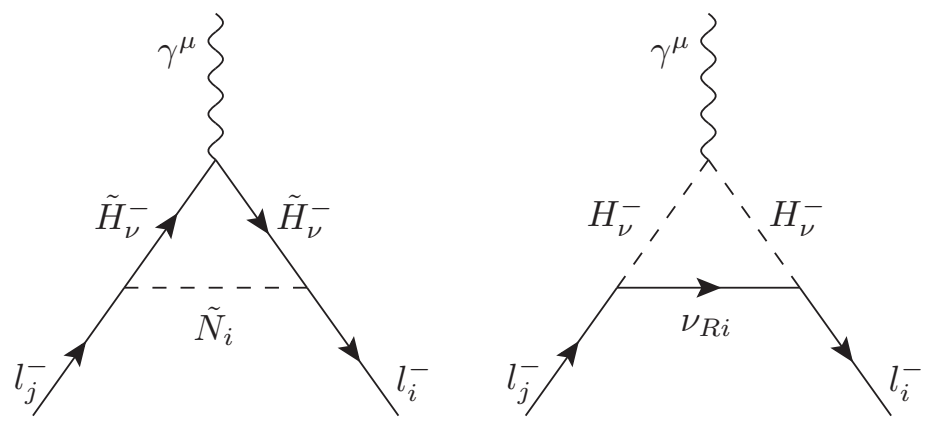

Figure 8. The Feynman diagrams for the lepton flavor violation corresponding to eq. (2.31).

For the muon decay, $\mu \rightarrow e \gamma$, the branching ratio is given by

$$
\operatorname{Br}(\mu \rightarrow e \gamma)=\frac{96 \pi^{3} \alpha}{G_{F}^{2}}\left(\left|\left(A_{2}\right)_{12}\right|^{2}+\left|\left(A_{3}\right)_{12}\right|^{2}\right) .
$$

The present bounds on the branching ratios of the LFV decays are [29, 30]

$$
\begin{aligned}
& \operatorname{Br}(\mu \rightarrow e \gamma)<5.7 \times 10^{-13} \quad(90 \% \text { C.L. }) \\
& \operatorname{Br}(\tau \rightarrow e \gamma)<3.3 \times 10^{-8} \quad(90 \% \text { C.L. }) \\
& \operatorname{Br}(\tau \rightarrow \mu \gamma)<4.4 \times 10^{-8} \quad(90 \% \text { C.L. }) .
\end{aligned}
$$

For our model with Yukawa interactions in eq. (2.2), from the diagrams given in figure 8, we obtain

$$
\begin{aligned}
& \left(A_{2}\right)_{\alpha \beta} \simeq \frac{1}{32 \pi^{2}}\left(\sum_{l} \frac{\left(S^{T} y_{\nu}\right)_{l \alpha}\left(S^{T} y_{\nu}\right)_{l \beta}}{M_{\tilde{H}_{\nu}^{-}}^{2}} F\left(\frac{M_{\tilde{N}_{l}}^{2}}{M_{\tilde{H}_{\nu}^{-}}^{2}}\right)-\sum_{k} \frac{\left(y_{\nu}\right)_{k \alpha}\left(y_{\nu}\right)_{k \beta}}{48 M_{H_{\nu}^{-}}^{2}}\right), \\
& \left(A_{3}\right)_{\alpha \beta}=0,
\end{aligned}
$$

with $F(x)$ being an auxiliary function. The experimental limits (2.34) give strong bounds on Yukawa couplings as well as masses of mediated particle, $M_{\tilde{N}_{k}}, M_{\tilde{H}_{\nu}^{-}}$and $M_{H_{\nu}^{ \pm}}$, as we will show. In fact, for $\mathcal{O}(100) \mathrm{GeV}$ masses of sneutrinos and the neutrinophilic chargino, we find the LFV decaying branching ratios are of $\mathcal{O}\left(10^{-6}\right)$, those are very large compared with current bounds (2.34). Since Yukawa couplings are fixed from the neutrino mass and are of the order of unity, the charged Higgs must be heavier than around $10 \mathrm{TeV}$ in the second term. On the other hand, for the first term, we have a possibility that one of sneutrinos and charged Higgsinos are relatively light in the case that $\tilde{y}=S^{T} y_{\nu}$ are suitably aligned by appropriate sneutrino mixings $\sigma_{i j}$ in such a way that the flavor-violating processes are suppressed enough. Such mixing angles can be found by requiring some off-diagonal components of $\tilde{y}=S^{T} y_{\nu}$ to be almost vanishing.

\subsection{Anomalous magnetic dipole moment of muon}

The muon anomalous magnetic dipole moment has $3.3 \sigma$ discrepancy between experimental data and the SM prediction as [31, 32]

$$
a_{\mu}(\mathrm{EXP})-a_{\mu}(\mathrm{SM})=(26.1 \pm 8.0) \times 10^{-10} .
$$


Thus, this discrepancy has been regarded as a hint and provided a motivation to investigate new physics beyond the standard model of particle physics.

$A_{2}$ and $A_{3}$ in eq. (2.31) contribute to the magnetic and electric dipole moment, respectively. The resultant electric and magnetic dipole moment of $l_{j}$ lepton are given as

$$
\begin{aligned}
& d_{l_{j}}=m_{l_{j}}\left(A_{3}\right)_{j j}, \\
& a_{l_{j}}=\frac{(g-2)_{l_{j}}}{2}=2 m_{l_{j}}^{2}\left(A_{2}\right)_{j j} .
\end{aligned}
$$

The additional contribution to the induced magnetic moment of muon in the supersymmetric neutrinophilic Higggs model with large Yukawa couplings is given by

$$
\delta a_{\mu}=2 m_{\mu}^{2} \frac{1}{32 \pi^{2}} \sum_{l} \frac{\left(S^{T} y_{\nu}\right)_{l \mu}\left(S^{T} y_{\nu}\right)_{l \mu}}{M_{\tilde{H}^{-}}^{2}} F\left(\frac{M_{\tilde{\nu}_{l}}^{2}}{M_{\tilde{H}^{-}}^{2}}\right),
$$

where we assumed Yukawa couplings are real and the negligible charged Higgs boson contribution is omitted. We might expect a large $g-2$ of the muon for light sneutrinos and the light $\tilde{H}_{\nu}$-like chargino because Yukawa coupling constants are $\mathcal{O}(1)$. However, as mentioned above, the experimental limits on the lepton flavor violation in eq. (2.34) are very stringent and we need a special mixing of sneutrinos.

\subsection{Compatibility in benchmarks}

As mentioned previous subsections, LFV constraints are very stringent. Indeed, for cases of vanishing lightest neutrino mass $m_{1}$ as in benchmark point 1 and 3 in table 1 , we could not find viable parameter sets. Thus, here we mention viable parameter sets based on the benchmark point 2 and 3 in the table 1.

At first, for the benchmark 2, we find that LFV constraints are avoided with the sneutrino mixing angles $\left(\sigma_{12}, \sigma_{23}, \sigma_{13}\right) \simeq(0.75,0.68,0.20)$, and the resultant $\tilde{y}$ is given by

$$
\tilde{y}=S^{T} y_{\nu} \simeq\left(\begin{array}{ccc}
1.18 & 0.06 & 0 \\
0 & 1.28 & 0 \\
0.05 & 0.24 & 1.31
\end{array}\right) \text {. }
$$

Then, the mass of the lightest RH sneutrino $\tilde{N}_{2}$ can be $10-100 \mathrm{GeV}$ without inducing a large LFV, while the mass of the other two $\tilde{N}_{1}$ and $\tilde{N}_{3}$ need to be $\mathcal{O}(1-10) \mathrm{TeV}$. Notice that with the definition of $S$ by eq. (2.10), we take the mass ordering of RH neutrinos as $M_{\tilde{N}_{2}}<M_{\tilde{N}_{1}}<M_{\tilde{N}_{3}}$. With this choice of sneutrino mixing angles, the muon decay width constraint discussed section 2.2 is avoided. Here, we can see that the coupling between the lightest RH sneutrino $\left(\tilde{N}_{2}\right)$ and the electron is negligibly small. This small coupling automatically suppresses the LEP mono-photon constraint from eq. (2.30).

In figure 9 , we show the viable sneutrino mixing angles from the constrains on the LFV processes for the benchmark 2. In those plots, we fixed one mixing angle $\sigma_{12}=0.75$, the neutrinophilic Higgsino mass $M_{\tilde{H}_{\nu}}=110 \mathrm{GeV}$, and the lightest sneutrino mass $M_{\tilde{N}_{2}}=$ $60 \mathrm{GeV}$. Heavy sneutrino masses, $\left(M_{\tilde{N}_{1}}, N_{\tilde{N}_{3}}\right)$ are taken from around $\mathrm{TeV}$ to $10 \mathrm{TeV}$. As the figures show, the constraint from $\mu \rightarrow e \gamma$ is most serious, and very small regions are 

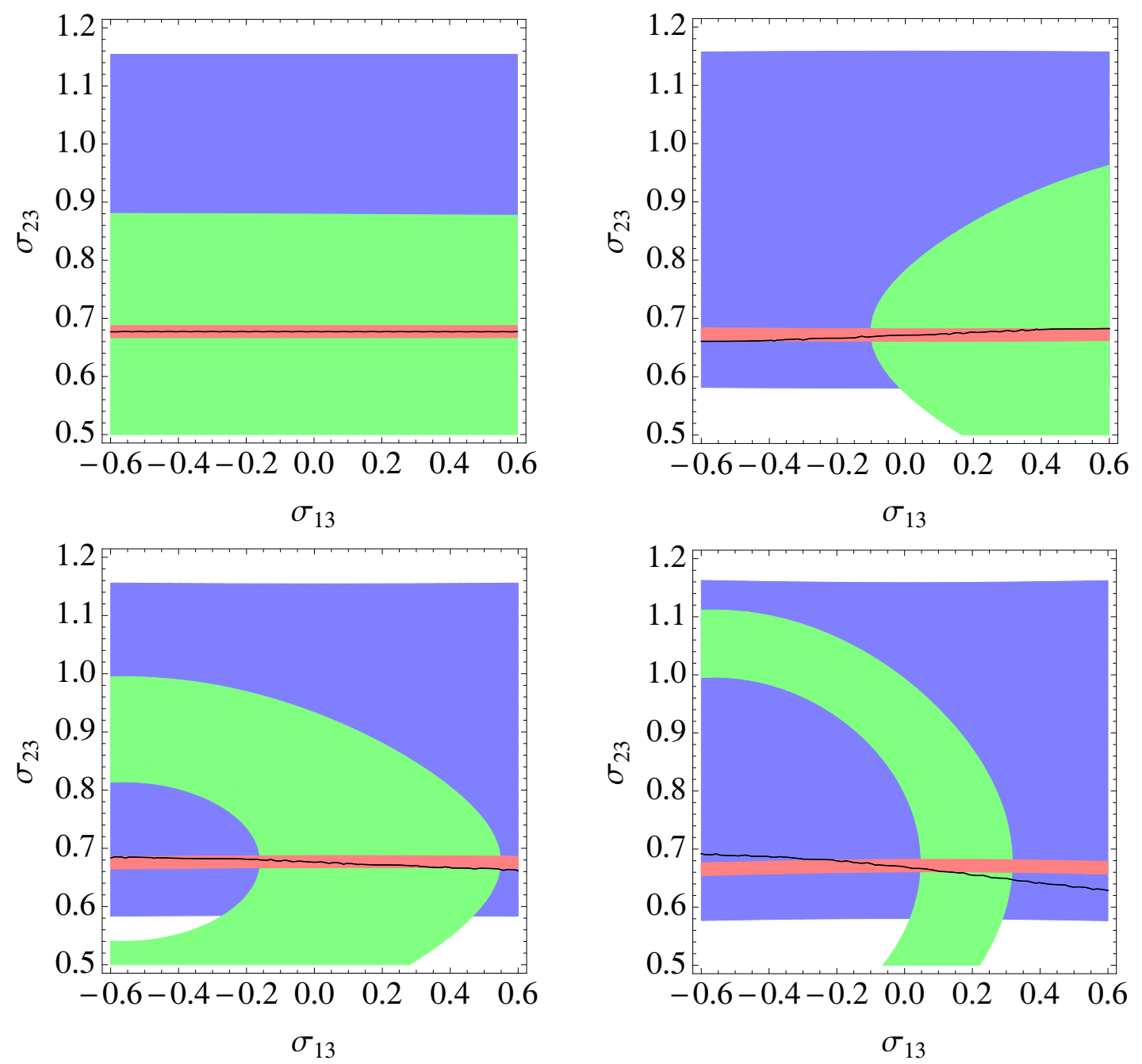

Figure 9. Allowed parameter space in the plane of the sneutrino mixing angles $\left(\sigma_{13}, \sigma_{23}\right)$ from the constraints on LFV for the benchmark 2. For all plots, we fixed $M_{\tilde{H}_{\nu}}=110 \mathrm{GeV}, M_{\tilde{N}_{2}}=$ $60 \mathrm{GeV}$ and $\sigma_{12}=0.75$. Top left corresponds to $\left(M_{\tilde{N}_{1}}, M_{\tilde{N}_{3}}\right)=(7 \mathrm{TeV}, 10 \mathrm{TeV})$. Top right, $(7 \mathrm{TeV}, 1 \mathrm{TeV})$. Bottom left, $(1 \mathrm{TeV}, 7 \mathrm{TeV})$. Bottom right, $(0.5 \mathrm{TeV}, 1 \mathrm{TeV})$. The black region is $\operatorname{Br}(\mu \rightarrow e \gamma)<5.7 \times 10^{-13}$, the red region is $\operatorname{Br}(\tau \rightarrow \mu \gamma)<4.4 \times 10^{-8}$, and the green region is $\operatorname{Br}(\tau \rightarrow e \gamma)<3.3 \times 10^{-8}$. The blue region denotes $\delta a_{\mu}>10^{-9}$.

allowed. Including the constrains from $\tau \rightarrow \mu \gamma$, we find that the value of $\sigma_{23}$ should be around 0.68 , which corresponds to $\left(S^{T} y_{\nu}\right)_{2 e} \approx 0$. The constraints do not restrict the value of $\sigma_{13}$ much for the case with two heavy sneutrino masses. In the allowed parameter space, the sizable $\delta a_{\mu}$ can be obtained as denoted by the blue colored region.

In figure 10, we show the viable parameter space for the benchmark 2 with contours of the contribution to the muon anomalous magnetic moment from eq. (2.39) in the plane of the mass of neutrinophilic Higgsino and the RH sneutrino mass $M_{\tilde{N}_{2}}$, which is the lightest supersymmetric particle. Here, we use $M_{\tilde{N}_{1}}=7 \mathrm{TeV}$ and $M_{\tilde{N}_{3}}=10 \mathrm{TeV}$ for reference. The region of Red and Orange color respectively show $1 \sigma$ and $2 \sigma$ range of eq. (2.36). The 


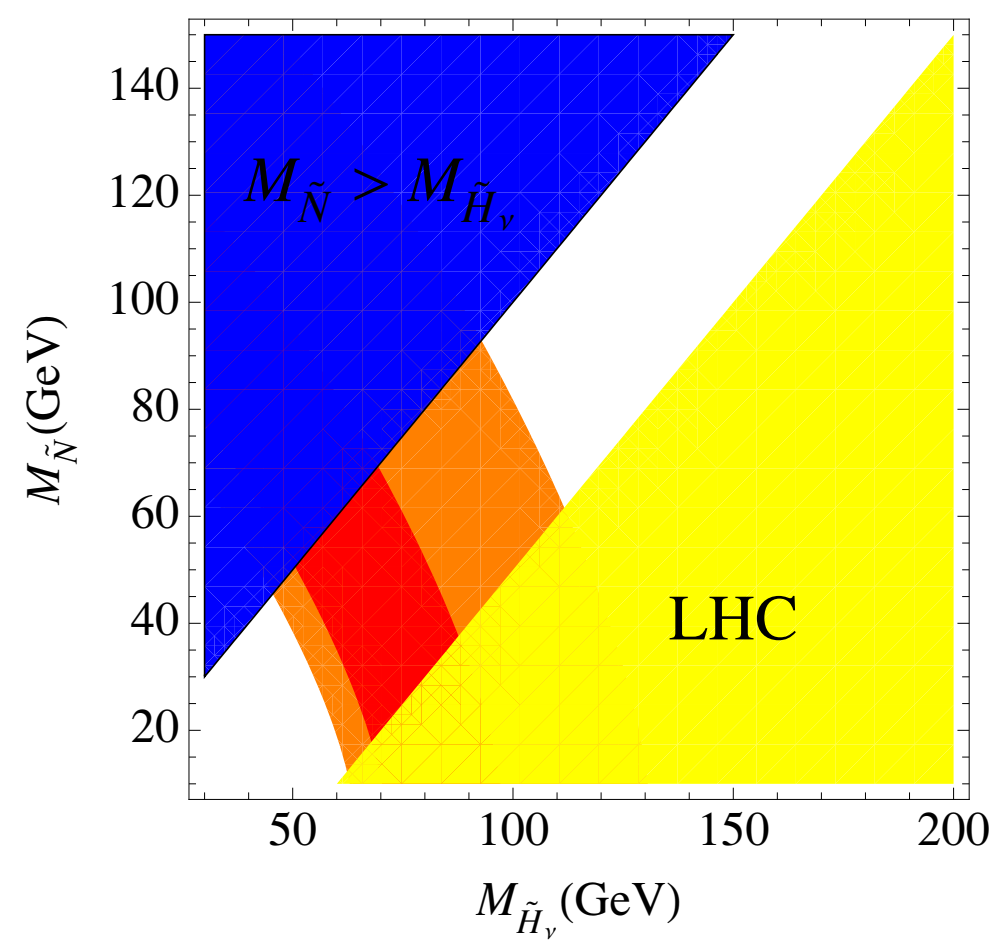

Figure 10. The viable parameter space for the benchmark 2 with tuned sneutrino mixing angles so that LFV processes are sufficiently suppressed. Contours show the contribution to the muon anomalous magnetic moment from eq. (2.39) in the plane of the mass of neutrinophilic Higgsino and the RH sneutrino mass $M_{\tilde{N}} \cdot 1 \sigma$ and $2 \sigma$ region of eq. (2.36) is shown with Red and Orange color respectively. In the blue region, $M_{\tilde{N}}>M_{\tilde{H}_{\nu}}$ is realized. The yellow region, where the mass splitting between sneutrino and chargino is too large, is constrained by the LHC results.

blue region corresponds $m_{\tilde{N}_{2}}>m_{\tilde{H}_{\nu}}$. The yellow region, where the mass splitting between sneutrino and chargino is too large, is constrained by the LHC results.

For the benchmark 3 , if the sneutrino mixing angles are $\left(\sigma_{12}, \sigma_{23}, \sigma_{13}\right) \simeq(0.75,0.93$, 0.02 ), the resultant $\tilde{y}$ is given by

$$
\tilde{y}=S^{T} y_{\nu} \simeq\left(\begin{array}{ccc}
1.20 & 0 & -0.25 \\
0 & 1.02 & 0 \\
0.21 & -0.33 & 1.05
\end{array}\right) .
$$

Then, the mass of the lightest RH sneutrino $\tilde{N}_{2}$ can be $10-100 \mathrm{GeV}$ without inducing a large LFV, while the mass of the other two $\tilde{N}_{1}$ and $\tilde{N}_{3}$ need to be $\mathcal{O}(1-10) \mathrm{TeV}$. In figure 11, we show the viable region with $M_{\tilde{N}_{1}}=7 \mathrm{TeV}$ and $M_{\tilde{N}_{3}}=8 \mathrm{TeV}$. Again, we take the mass ordering of RH sneutrinos as $M_{\tilde{N}_{2}}<M_{\tilde{N}_{1}}<M_{\tilde{N}_{3}}$.

\section{Cosmological constraints}

\subsection{Dark radiation}

$\mathrm{RH}$ component of neutrinos could contribute to the additional relativistic degrees of freedom in the early Universe, which is constrained by big bang nucleosysthesis and cosmic 


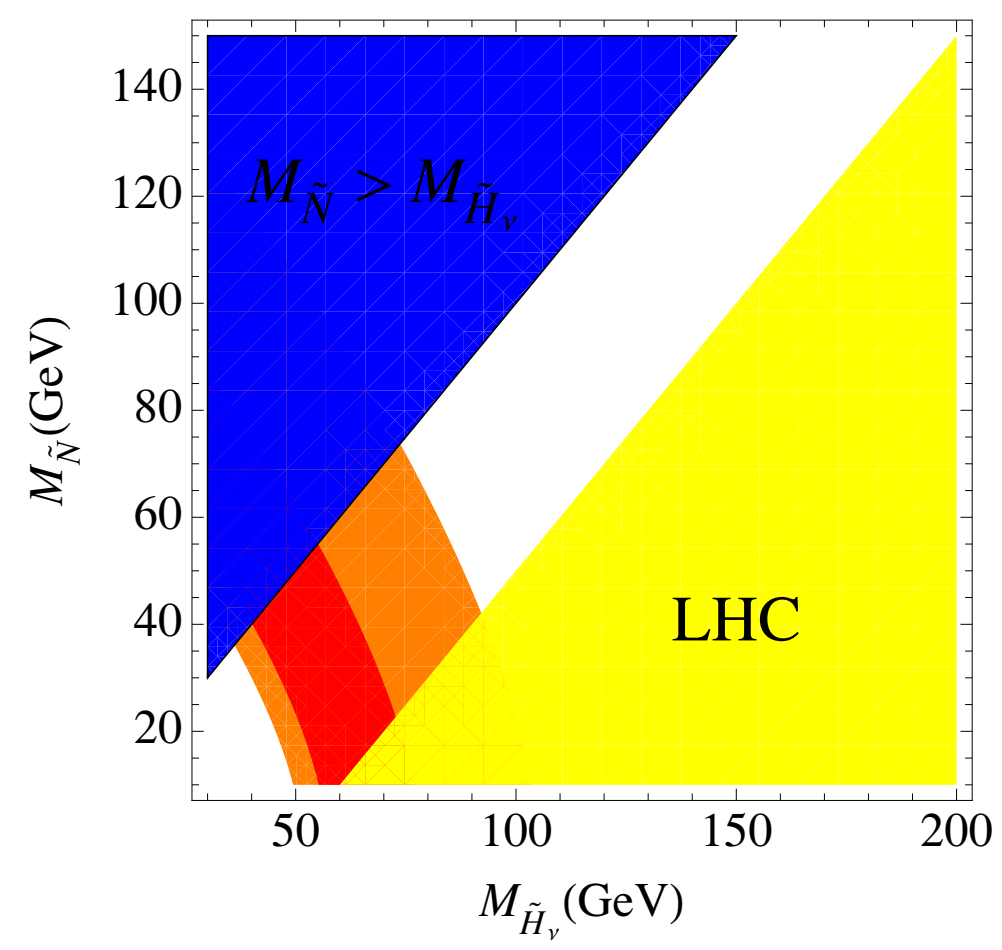

Figure 11. Same as figure 10 for the benchmark 3.

microwave background radiation observation as $\Delta N_{\text {eff }} \lesssim 0.57$. In our model, $\mathrm{RH}$ neutrinos could be in thermal equilibrium due to the scatterings with charged leptons (left-handed neutrinos) through neutrinophilic charged (neutral) Higgs bosons via the Yukawa interaction in eq. (2.2).

In order to suppress $\Delta N_{\text {eff }}$ enough, RH neutrinos should have decoupled from the thermal bath much before the quark-hadron phase transition which takes place at the cosmic temperature $T_{c} \simeq 200 \mathrm{MeV}$.

\subsection{1 $l^{-} l^{+} \longleftrightarrow \nu_{R} \overline{\nu_{R}}$}

The scatterings between charged lepton and $\mathrm{RH}$ neutrinos are mediated by the neutrinophilic charged Higgs boson $H_{\nu}^{ \pm}$. We obtain

$$
\int \overline{|\mathcal{M}|^{2}} d \mathrm{LIPS} \simeq \frac{1}{8 \pi} \frac{\left|y_{\nu} y_{\nu}\right|^{2}}{M_{H_{\nu}^{+}}^{4}} \frac{s^{2}}{12}
$$

with $s$ being the energy at the center of mass frame. Taking thermal average, we find

$$
\langle\sigma v\rangle \simeq \frac{\left|y_{\nu} y_{\nu}\right|^{2}}{32 \pi M_{H_{\nu}^{+}}^{4}} T^{2}
$$




\subsection{2 $\nu_{L} \overline{\nu_{L}} \longleftrightarrow \nu_{R} \overline{\nu_{R}}$}

Similarly, the thermal scattering cross section between left-handed and right-handed components of neutrinos via $H_{\nu}^{0}$ and $A_{\nu}^{0}$ is estimated as

$$
\langle\sigma v\rangle \simeq \frac{\left|y_{\nu} y_{\nu}\right|^{2}}{32 \pi} \frac{1}{4}\left(\frac{1}{M_{H_{\nu}^{0}}^{2}}+\frac{1}{M_{A_{\nu}^{0}}^{2}}\right)^{2} T^{2} .
$$

\subsubsection{Decoupling condition}

The decoupling condition of $\mathrm{RH}$ neutrino at the quark-hadron transition epoch is expressed as

$$
\left.\langle\sigma v\rangle n\right|_{T_{\mathrm{QH}}}<\left.H\right|_{T_{\mathrm{QH}}}
$$

which is rewritten as

$$
\frac{\left|y_{\nu} y_{\nu}\right|^{2}}{M_{H^{+}}^{4}}+\frac{\left|y_{\nu} y_{\nu}\right|^{2}}{4}\left(\frac{1}{M_{H_{\nu}^{0}}^{2}}+\frac{1}{M_{A_{\nu}^{0}}^{2}}\right)^{2}<\sqrt{\frac{g_{*}}{90}} \frac{64 \pi^{4}}{3 \zeta(3) T_{\mathrm{QH}}^{3} M_{P}}=\frac{1}{(3.3 \mathrm{TeV})^{4}}\left(\frac{0.1 \mathrm{GeV}}{T_{\mathrm{QH}}}\right)^{3} .
$$

Therefore the neutrinophilic Higgs must be heavier than around $3 \mathrm{TeV}$ for order of unity Yukawa couplings. We note that the similar bound has already been obtained but $H_{\nu}^{0}$ and $A_{\nu}^{0}$ contributions were missing in refs. [11, 23, 24]. Here we have re-estimated and corrected it.

\section{Dark matter}

The lightest RH sneutrino is stable when R-parity is preserved and can be a good candidate for dark matter. The possibility in the neutrinophilic Higgs model was suggested in refs. $[9,10]$ by two of the present authors. In this section we generalise the previous results considering the benchmark points in the previous section and examine the cosmological and astrophysical phenomenon.

\subsection{Relic density of dark matter}

Due to the large Yukawa coupling in eq. (2.2), the RH sneutrino interacts with fermions and Higgsinos efficiently so that they could be in the thermal equilibrium at high temperature. In the rapidly expanding early Universe, those $\mathrm{RH}$ sneutrinos decouple from the thermal plasma and the comoving abundance is conserved after that. The relic density of WIMPs is determined by the annihilation cross section which determines the freeze-out temperature of DM. However, for complex fields, there might be the non-vanishing DM asymmetry. With a large DM asymmetry, the final relic density of DM may depend on the annihilation cross section and the DM asymmetry [33-39]. This is the case for light RH sneutrino DM in our scenario.

The annihilation cross section of RH sneutrino DM is dominantly determined by the annihilations into the leptons, that is given in partial wave expansion method by $[9,10]$

$$
\langle\sigma v\rangle_{f \bar{f}}=\sum_{f}\left(\frac{y_{\nu}^{4}}{16 \pi} \frac{m_{f}^{2}}{\left(M_{\tilde{N}}^{2}+M_{\tilde{H}_{\nu}}^{2}\right)^{2}}+\frac{y_{\nu}^{4}}{8 \pi} \frac{M_{\tilde{N}}^{2}}{\left(M_{\tilde{N}}^{2}+M_{\tilde{H}_{\nu}}^{2}\right)^{2}} \frac{T}{M_{\tilde{N}}}+\ldots\right) .
$$


There is another subdominant contribution from the induced annihilation into photons,

$$
\langle\sigma v\rangle_{2 \gamma}=\frac{|M|_{2 \gamma}^{2}}{32 \pi M_{\tilde{N}}^{2}} \simeq \frac{\alpha_{\mathrm{em}}^{2}}{8 \pi^{3}} \frac{y_{\nu}^{4}\left(A_{\nu}^{2}+\mu^{\prime 2}\right)^{2}}{M_{\tilde{l}}^{4}} \frac{4}{M_{\tilde{N}}^{2}},
$$

where we used the approximation of $M_{H_{\nu}}=M_{H_{\nu}^{\prime}}=M_{\tilde{l}}$ for simplicity and the soft term $\mathcal{L}=y_{\nu} A_{\nu} \tilde{L} H_{\nu} \tilde{N}+$ h.c.. In fact, it gives small subdominant contribution to determine the relics density of DM in our consideration with $y_{\nu}^{4}\left(A_{\nu}^{2}+\mu^{\prime 2}\right)^{2} \simeq M_{\tilde{l}}^{4}$.

Since the RH sneutrinos were in the thermal equilibrium, the asymmetry could be generated from non-zero baryon asymmetry during the sphaleron process. The asymmetry of RH sneutrinos will depend on the specific model of baryogenesis, mass spectrum and the electroweak phase transition. In the simple case, the leptonic asymmetry is expected to be the order of baryon asymmetry, as $10^{-10}$ [11]. In this paper, in order to see the asymmetry dependence, we treat it as a free parameter taking a value of a certain range.

For the WIMP with a non-vanishing asymmetry, the resulting relic density can be estimated by [40, 41]

$$
\Omega_{\mathrm{DM}} h^{2}=2.8 \times 10^{8} \frac{m_{\mathrm{DM}}}{\mathrm{GeV}}\left(Y_{\mathrm{DM}}+Y_{\overline{\mathrm{DM}}}\right)
$$

Here

$$
Y_{\mathrm{DM}}+Y_{\overline{\mathrm{DM}}}=\frac{C}{1-\exp \left[-C \lambda J\left[x_{F}\right]\right]}+\frac{C}{\exp \left[C \lambda J\left[\bar{x}_{F}\right]\right]-1}
$$

with

$$
\begin{aligned}
\lambda & =4 \pi \sqrt{\frac{g_{*}}{90}} M_{P} m_{\mathrm{DM}}, \\
J\left[x_{F}\right] & =\int_{x_{F}}^{\infty}\langle\sigma v\rangle x^{-2} d x, \\
x & =m_{\mathrm{DM}} / T,
\end{aligned}
$$

where $x_{F}\left(\bar{x}_{F}\right)$ denotes the value of $x$ at the "freeze out" time of (anti-)dark matter particle. The asymmetry of dark matter is given by

$$
C=\frac{n_{\mathrm{DM}}-n_{\overline{\mathrm{DM}}}}{s} .
$$

In the figure 12 we show the contour plot of the corresponding DM asymmetry to give the correct relic density of DM. For a given asymmetries $C=\left(5 \times 10^{-11}, 10^{-11}, 5 \times\right.$ $\left.10^{-12}, 3 \times 10^{-12}, 10^{-12}\right)$, the required amount of DM is obtained on the corresponding (thick solid, solid, dashed, dotted, solid) lines in the plane of DM mass and Higgsino mass.

For a smaller Higgsino mass, the annihilation cross section is large enough to annihilate the DM-anti DM pairs and only the asymmetry remains. In this case, the abundance is determined by the asymmetry and the relic density is proportional to the DM mass and the asymmetry. We can see this for the Higgsino mass between $80 \mathrm{GeV}$ and $140 \mathrm{GeV}$, where the contour lines are parallel and horizontal, and the mass of dark matter is inversely proportional to the asymmetry. For large Higgsino mass, the annihilation cross section is too small to annihilate all anti DM. In this case, the relic density is determined by the 


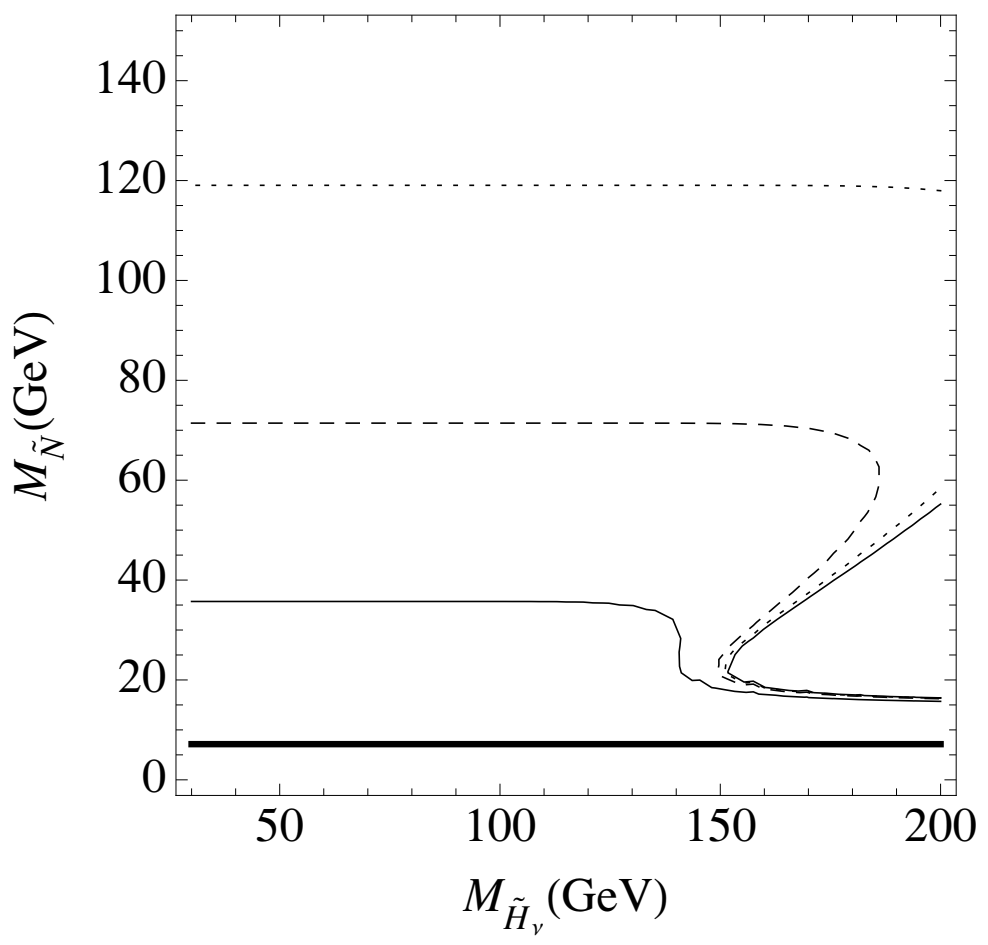

Figure 12. The contour plot of the DM asymmetry $C=\left(5 \times 10^{-11}, 10^{-11}, 5 \times 10^{-12}, 3 \times 10^{-12}\right.$, $10^{-12}$ ) with (thick solid, solid, dashed, dotted, solid lines) given in eq. (4.8), to give correct relic density for DM in the plane of its mass and Higgsino mass.

annihilation cross section as the usual WIMP, being independent from the asymmetry. That can be seen, in the figure, around Higgsino mass larger than $150 \mathrm{GeV}$ and RH sneutrino mass smaller than $60 \mathrm{GeV}$, where contour lines are not horizontal.

Compared to figure 10 and 11, the region compatible to explain the muon anomalous magnetic moment corresponds to Higgsino mass between $40 \mathrm{GeV}$ and $120 \mathrm{GeV}$ and the DM relic density is dominantly determined by the DM asymmetry larger than around $C=5 \times 10^{-12}$.

\subsection{Dark matter scattering cross section with a nucleon}

An elastic scattering of RH sneutrino with nuclei may induce a signal in the direct detection experiments. The most relevant process for our sneutrino DM is through the $Z$-boson exchange even this is not $\mathrm{LH}$ but $\mathrm{RH}[9,10]$, in contrast with most of other $\mathrm{RH}$ sneutrino DM models where the Higgs bosons exchange is dominant [42-50]. The DM-DM- $Z$ vertex is induced by one-loop digram involving neutrinophilic Higgs boson. Because of the $Z$ boson exchange, DM mostly scatters with only neutrons rather than protons. In figure 13, we show the scattering cross section with a neutron for $\left(S^{T} y_{\nu}\right) A_{\nu} / M_{H_{\nu}}=0.1,1,2,10$ from the bottom to the top, and the current bounds by direct detection experiments, especially, the LUX experiment [51] with the black thick line. The experimental bound shown in the figure is re-estimated for our DM which scatters off with only neutrons. Here $A_{\nu}$ is the trilinear soft coupling defined after eq. (4.2). 


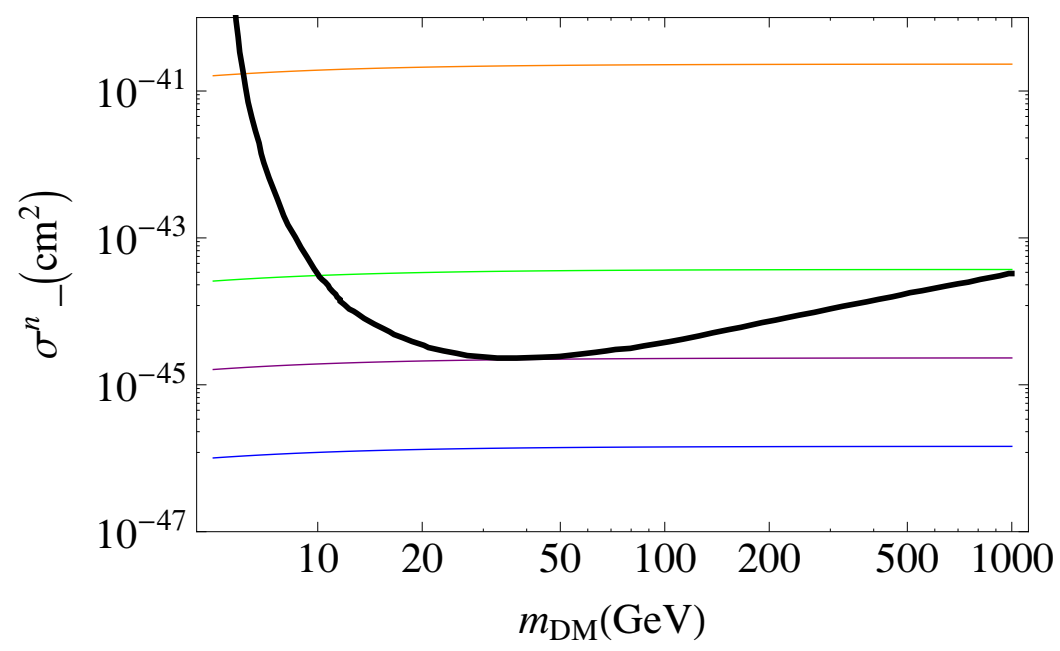

Figure 13. The scattering cross section between the lightest $\mathrm{RH}$ sneutrino and a neutron for $\left(S^{T} y_{\nu}\right) A_{\nu} / M_{H_{\nu}}=0.5,1,2,10$ from the bottom to the top. The current LUX bound on it is also shown with the black thick line.

\subsection{Indirect signal from sneutrino dark matter}

For the asymmetric DM, their annihilation in the galaxy is negligible. However, they can scatter off the cosmic rays and produce secondary particles such as gamma-ray or neutrinos [52]. Since we are considering the large Yukawa couplings, the indirect signature might be very promising or even harmful. However, in our benchmark point 2 and 3 , the Yukawa coupling between the lightest RH sneutrino $\left(N_{2}\right)$ and the electron is quite negligible as a consequence of suppressing LFV and therefore the indirect signature is also very suppressed.

For symmetric DM case with the heavy $\tilde{H}_{\nu}^{ \pm}$, the DM annihilation signal can be seen most likely as a gamma-ray line because annihilation into a fermion pair is helicity suppressed [9].

\subsection{Decay of cosmic neutrino background}

As the muon can decay to the electron through the charged neutrinophilic Higgs(ino), and sneutrino loops, the heavier neutrino can decay to the lighter one through similar diagrams. Assuming that the mass of lighter one is much smaller than that of the heavier one, the decay rate of neutrino is

$$
\Gamma\left(\nu_{j} \rightarrow \nu_{i}+\gamma\right)=\frac{e^{2}}{4 \pi} m_{\nu_{j}}^{5}\left(\left|\left(A_{2}^{\nu}\right)_{i j}\right|^{2}+\left|\left(A_{3}^{\nu}\right)_{i j}\right|^{2}\right)
$$

where

$$
\begin{aligned}
& \left(A_{2}^{\nu}\right)_{i j} \simeq \frac{1}{32 \pi^{2}}\left(\sum_{\alpha} \frac{\left(y_{\nu}\right)_{i \alpha}\left(y_{\nu}\right)_{j \alpha}}{M_{\tilde{H}_{\nu}^{-}}^{2}} F\left(\frac{M_{\tilde{l}_{\alpha}}^{2}}{M_{\tilde{H}_{\nu}^{-}}^{2}}\right)-\sum_{\beta} \frac{\left(y_{\nu}\right)_{i \beta}\left(y_{\nu}\right)_{j \beta}}{48 M_{H_{\nu}^{-}}^{2}}\right), \\
& \left(A_{3}^{\nu}\right)_{i j}=0 .
\end{aligned}
$$


Although the GIM mechanism is not applied, so there is no suppression by the mass of charged leptons, the suppression by small neutrino mass as 5th powers is enough to satisfy the present constraint on the life-time of the neutrinos, which is

$$
\tau_{\nu}>10^{12} \mathrm{yrs},
$$

from the analysis of the cosmic infrared background [53].

\section{Conclusion}

We have studied an extended supersymmetric model where neutrinos are Dirac particle and those masses are given by large neutrino Yukawa couplings and a small VEV of the neutrinophilic Higgs field. Provided the lightest RH sneutrino is LSP as the dark matter candidate, we have examined various aspects of the model with Dirac Yukawa couplings of the order of unity.

By only considering the muon decay width, it turns out that the neutrinophilic Higgs bosons must be heavier than several hundreds $\mathrm{GeV}$ and some supersymmetric particles among RH sneutrinos and neutrinophilic Higgsino need to be heavier than several hundreds $\mathrm{GeV}$. In fact, we have found that the neutrinophilic Higgsino and one of the RH sneutrino can be relatively light of the order of $100 \mathrm{GeV}$ if the other two $\mathrm{RH}$ neutrinos are heavy enough. The current collider experiment, most importantly the LHC, constraints require a viable parameter space; $\tilde{H}_{\nu}^{ \pm}$is heavy enough, or the mass difference of $\tilde{H}_{\nu}^{ \pm}$and the lightest $\mathrm{RH}$ sneutrino is smaller than about $50 \mathrm{GeV}$, according to ref. [28]. The LEP constrains the $\tilde{H}_{\nu}^{ \pm}-\tilde{N}_{\mathrm{DM}}-e$ coupling to be less than about 0.6 .

For general mixing of $\mathrm{RH}$ sneutrinos, due to the large flavor mixings of neutrino sector, the lepton flavor violating processes induced through neutrino Yukawa interactions are also typically as large as $10^{-6}$ in the decay branching ratio, with new particles of $\mathcal{O}(100) \mathrm{GeV}$ mass. Only with appropriately tuned RH sneutrino mixings, we can avoid the LFVs.

With the chosen parameters, we found that the deviation of the muon $g-2$ can be explained with a relatively large lightest neutrino mass around $m_{1} \simeq 0.05 \mathrm{eV}$ and the lightest RH sneutrino and Higgsino mass, $M_{\tilde{N}}=10-100 \mathrm{GeV}$ and $M_{\tilde{H}_{\nu}}=60-160 \mathrm{GeV}$ respectively. In other words, if the muon $g-2$ is explained in this model, then $m_{1}$ can not be so small. As a result of tuned RH sneutrino mixings, the $\tilde{H}_{\nu}^{ \pm}-\tilde{N}_{\mathrm{DM}}-e$ coupling is almost vanishing, which means the LEP data does not significantly constrain this model and the international linear collider also would not be able to produce a mono-photon signal, while the $\tilde{H}_{\nu}^{ \pm}-\tilde{N}_{\mathrm{DM}}-\mu$ coupling is about unity. A muon collider can easily test this model if it will be indeed constructed, as the Fermilab plans [54].

In this muon $g-2$ favored parameter region, the DM relic density is explained by $\mathrm{RH}$ sneutrino with the asymmetry of $C \sim 5 \times 10^{-12}$. If we do not mind the deviation of the muon $g-2$, RH sneutrino dark matter could be an usual WIMP with heavier $\tilde{H}_{\nu}^{ \pm}$.

\section{Acknowledgments}

K.-Y.C. appreciates Asia Pacific Center for Theoretical Physics for the support to the Topical Research Program. K.-Y.C. was supported by the Basic Science Research Program 
through the National Research Foundation of Korea (NRF) funded by the Ministry of Education, Science and Technology Grant No. 2011-0011083. O.S. was supported in part by the Grant-in-Aid for Scientific Research on Innovative Areas No. 26105514 from the Ministry of Education, Culture, Sports, Science and Technology in Japan. C.S.S. is supported in part by DOE grants doe-sc0010008, DOE-ARRA-SC0003883, and DOE-DE-SC0007897.

\section{A Formula for the muon $g-2$}

For the Lagrangian

$$
\mathcal{L}=\bar{L}\left(\lambda_{v}+i \lambda_{a} \gamma_{5}\right) f S+\text { h.c. },
$$

where $f$ is charged fermion, the anomalous magnetic dipole moment of the charged lepton $L$ with charge $Q$ is given by $[55,56]$

$$
\delta a_{L}=\frac{m_{L}}{8 \pi^{2}} \int_{0}^{1} d z \frac{\left(\left|\lambda_{v}\right|^{2}+\left|\lambda_{a}\right|^{2}\right) z(1-z)^{2} m_{L}+\left(\left|\lambda_{v}\right|^{2}-\left|\lambda_{a}\right|^{2}\right)(1-z)^{2} m_{f}}{-z(1-z) m_{L}^{2}+(1-z) m_{f}^{2}+z m_{s}^{2}} .
$$

For neutrinophilic Higgs model with Lagrangian

$$
\mathcal{L}=y \tilde{N} \bar{L} P_{R} \tilde{H}_{\nu}+\text { h.c. }
$$

we obtain

$$
\begin{aligned}
\delta a_{L} & =\frac{|y|^{2} m_{L}^{2}}{16 \pi^{2}} \int_{0}^{1} d z \frac{z(1-z)^{2}}{-z(1-z) m_{L}^{2}+(1-z) m_{f}^{2}+z m_{s}^{2}} \\
& \simeq \frac{|y|^{2} m_{L}^{2}}{16 \pi^{2} m_{f}^{2}} \int_{0}^{1} d z \frac{z(1-z)^{2}}{1-z+z\left(m_{s}^{2} / m_{f}^{2}\right)} .
\end{aligned}
$$

\section{B Auxiliary functions}

$$
\begin{aligned}
F(x) & =\int_{0}^{1} \frac{z(1-z)^{2}}{(1-z)+z x} \\
= & \frac{1-6 x+3 x^{2}+2 x^{3}-6 x^{2} \ln (x)}{6(x-1)^{4}}, \\
F_{2}(x, y)= & \frac{1}{x^{2}-y^{2}} \ln \left(\frac{x^{2}}{y^{2}}\right) \\
F_{3}(x, y, z, w)= & \frac{x^{4}\left(z^{2}-w^{2}\right) \log \left(-x^{2}\right)+w^{4}\left(x^{2}-z^{2}\right) \log \left(-w^{2}\right)+z^{4}\left(w^{2}-x^{2}\right) \log \left(-z^{2}\right)}{\left(x^{2}-y^{2}\right)\left(z^{2}-w^{2}\right)\left(x^{2}-z^{2}\right)\left(x^{2}-w^{2}\right)} \\
& -\frac{y^{4}\left(z^{2}-w^{2}\right) \log \left(-y^{2}\right)+w^{4}\left(y^{2}-z^{2}\right) \log \left(-w^{2}\right)+z^{4}\left(w^{2}-y^{2}\right) \log \left(-z^{2}\right)}{\left(x^{2}-y^{2}\right)\left(z^{2}-w^{2}\right)\left(z^{2}-y^{2}\right)\left(w^{2}-y^{2}\right)} .
\end{aligned}
$$

Open Access. This article is distributed under the terms of the Creative Commons Attribution License (CC-BY 4.0), which permits any use, distribution and reproduction in any medium, provided the original author(s) and source are credited. 


\section{References}

[1] ATLAS collaboration, Observation of a new particle in the search for the standard model Higgs boson with the ATLAS detector at the LHC, Phys. Lett. B 716 (2012) 1 [arXiv: 1207.7214] [INSPIRE].

[2] CMS collaboration, Observation of a new boson at a mass of $125 \mathrm{GeV}$ with the CMS experiment at the LHC, Phys. Lett. B 716 (2012) 30 [arXiv:1207.7235] [INSPIRE].

[3] T. Yanagida, Horizontal symmetry and masses of neutrinos, in Proceedings of the Workshop on the Baryon Number of the Universe and Unified Theories, Tsukuba Japan, 13-14 Feb 1979, A. Sawada and A. Sugamoto eds., pg. 95 [InSPIRE].

[4] M. Gell-Mann, P. Ramond and R. Slansky, Complex spinors and unified theories, in Proceedings of the Supergravity Workshop, Stony Brook, New York U.S.A., 27-28 Sep 1979, P. Van Nieuwenhuizen and D.Z. Freedman eds., pg. 315 [arXiv:1306.4669] [INSPIRE].

[5] R.N. Mohapatra and G. Senjanović, Neutrino mass and spontaneous parity violation, Phys. Rev. Lett. 44 (1980) 912 [InSPIRE].

[6] E. Ma, Naturally small seesaw neutrino mass with no new physics beyond the TeV scale, Phys. Rev. Lett. 86 (2001) 2502 [hep-ph/0011121] [INSPIRE].

[7] F. Wang, W. Wang and J.M. Yang, Split two-Higgs-doublet model and neutrino condensation, Europhys. Lett. 76 (2006) 388 [hep-ph/0601018] [INSPIRE].

[8] S. Gabriel and S. Nandi, A new two Higgs doublet model, Phys. Lett. B 655 (2007) 141 [hep-ph/0610253] [INSPIRE].

[9] K.-Y. Choi and O. Seto, A Dirac right-handed sneutrino dark matter and its signature in the gamma-ray lines, Phys. Rev. D 86 (2012) 043515 [Erratum ibid. D 86 (2012) 089904] [arXiv: 1205.3276] [INSPIRE].

[10] K.-Y. Choi and O. Seto, Light Dirac right-handed sneutrino dark matter, Phys. Rev. D 88 (2013) 035005 [arXiv: 1305.4322] [INSPIRE].

[11] P. Mitropoulos, Right-handed sneutrinos as asymmetric DM and neutrino masses from neutrinophilic Higgs bosons, JCAP 11 (2013) 008 [arXiv:1307.2823] [INSPIRE].

[12] N. Haba and O. Seto, Low scale thermal leptogenesis in neutrinophilic Higgs doublet models, Prog. Theor. Phys. 125 (2011) 1155 [arXiv:1102.2889] [InSPIRE].

[13] N. Haba and O. Seto, Thermal leptogenesis in a supersymmetric neutrinophilic Higgs model, Phys. Rev. D 84 (2011) 103524 [arXiv:1106.5354] [InSPIRE].

[14] N. Haba, O. Seto and Y. Yamaguchi, Resonant leptogenesis with mild degeneracy, Phys. Rev. D 87 (2013) 123540 [arXiv: 1305.2484] [INSPIRE].

[15] Particle Data Group collaboration, J. Beringer et al., Review of particle physics, Phys. Rev. D 86 (2012) 010001 [INSPIRE].

[16] Planck collaboration, P.A.R. Ade et al., Planck 2013 results. XVI. Cosmological parameters, arXiv:1303.5076 [INSPIRE].

[17] T. Fukuyama and K. Tsumura, Detecting Majorana nature of neutrinos in muon decay, arXiv:0809.5221 [INSPIRE].

[18] W. Fetscher, H.J. Gerber and K.F. Johnson, Muon decay: complete determination of the interaction and comparison with the standard model, Phys. Lett. B 173 (1986) 102 [INSPIRE].

[19] P. Herczeg, The nature of neutrinos in muon decay and physics beyond the standard model, Los Alamos Sci. 25 (1997) 128 [INSPIRE]. 
[20] W.J. Marciano, Fermi constants and 'new physics', Phys. Rev. D 60 (1999) 093006 [hep-ph/9903451] [INSPIRE].

[21] J. Erler and M.J. Ramsey-Musolf, Low energy tests of the weak interaction, Prog. Part. Nucl. Phys. 54 (2005) 351 [hep-ph/0404291] [InSPIRE].

[22] CHARM-II collaboration, P. Vilain et al., A precise measurement of the cross-section of the inverse muon decay $\nu_{\mu}+e^{-} \rightarrow \mu^{-}+\nu_{e}$, Phys. Lett. B 364 (1995) 121 [INSPIRE].

[23] S.M. Davidson and H.E. Logan, Dirac neutrinos from a second Higgs doublet, Phys. Rev. D 80 (2009) 095008 [arXiv:0906.3335] [InSPIRE].

[24] S.M. Davidson and H.E. Logan, LHC phenomenology of a two-Higgs-doublet neutrino mass model, Phys. Rev. D 82 (2010) 115031 [arXiv: 1009.4413] [INSPIRE].

[25] N. Haba and K. Tsumura, $\nu$-two Higgs doublet model and its collider phenomenology, JHEP 06 (2011) 068 [arXiv:1105.1409] [INSPIRE].

[26] LEP SUSY Working Group, ALEPH, DELPHI, L3, OPAL experiments, Notes LEPSUSYWG/01-03.1 and 04-01.1, http://lepsusy.web.cern.ch/lepsusy/Welcome.html.

[27] P.J. Fox, R. Harnik, J. Kopp and Y. Tsai, LEP shines light on dark matter, Phys. Rev. D 84 (2011) 014028 [arXiv: 1103.0240] [InSPIRE].

[28] J. Guo, Z. Kang, J. Li, T. Li and Y. Liu, Simplified supersymmetry with sneutrino LSP at $8 \mathrm{TeV}$ LHC, arXiv:1312.2821 [INSPIRE].

[29] MEG collaboration, J. Adam et al., New constraint on the existence of the $\mu^{+} \rightarrow e^{+} \gamma$ decay, Phys. Rev. Lett. 110 (2013) 201801 [arXiv:1303.0754] [INSPIRE].

[30] BABAR collaboration, B. Aubert et al., Searches for lepton flavor violation in the decays $\tau^{ \pm} \rightarrow e^{ \pm} \gamma$ and $\tau^{ \pm} \rightarrow \mu^{ \pm} \gamma$, Phys. Rev. Lett. 104 (2010) 021802 [arXiv:0908.2381] [INSPIRE].

[31] Muon G-2 collaboration, G.W. Bennett et al., Final report of the E821 muon anomalous magnetic moment measurement at BNL, Phys. Rev. D 73 (2006) 072003 [hep-ex/0602035] [INSPIRE].

[32] K. Hagiwara, R. Liao, A.D. Martin, D. Nomura and T. Teubner, $(g-2)_{\mu}$ and $\alpha\left(M_{Z}^{2}\right)$ re-evaluated using new precise data, J. Phys. G 38 (2011) 085003 [arXiv:1105.3149] [INSPIRE].

[33] S.M. Barr, R.S. Chivukula and E. Farhi, Electroweak fermion number violation and the production of stable particles in the early universe, Phys. Lett. B 241 (1990) 387 [InSPIRE].

[34] S.M. Barr, Baryogenesis, sphalerons and the cogeneration of dark matter, Phys. Rev. D 44 (1991) 3062 [INSPIRE].

[35] D.B. Kaplan, A single explanation for both the baryon and dark matter densities, Phys. Rev. Lett. 68 (1992) 741 [INSPIRE].

[36] S.D. Thomas, Baryons and dark matter from the late decay of a supersymmetric condensate, Phys. Lett. B 356 (1995) 256 [hep-ph/9506274] [INSPIRE].

[37] D. Hooper, J. March-Russell and S.M. West, Asymmetric sneutrino dark matter and the $\Omega_{b} / \Omega_{\mathrm{DM}}$ puzzle, Phys. Lett. B 605 (2005) 228 [hep-ph/0410114] [INSPIRE].

[38] R. Kitano and I. Low, Dark matter from baryon asymmetry, Phys. Rev. D 71 (2005) 023510 [hep-ph/0411133] [INSPIRE].

[39] D.E. Kaplan, M.A. Luty and K.M. Zurek, Asymmetric dark matter, Phys. Rev. D 79 (2009) 115016 [arXiv:0901.4117] [INSPIRE]. 
[40] M.L. Graesser, I.M. Shoemaker and L. Vecchi, Asymmetric WIMP dark matter, JHEP 10 (2011) 110 [arXiv:1103.2771] [INSPIRE].

[41] H. Iminniyaz, M. Drees and X. Chen, Relic abundance of asymmetric dark matter, JCAP 07 (2011) 003 [arXiv: 1104.5548] [INSPIRE].

[42] D.G. Cerdeño, C. Muñoz and O. Seto, Right-handed sneutrino as thermal dark matter, Phys. Rev. D 79 (2009) 023510 [arXiv: 0807. 3029] [INSPIRE].

[43] D.G. Cerdeño and O. Seto, Right-handed sneutrino dark matter in the NMSSM, JCAP 08 (2009) 032 [arXiv: 0903.4677] [INSPIRE].

[44] D.G. Cerdeño, J.-H. Huh, M. Peiro and O. Seto, Very light right-handed sneutrino dark matter in the NMSSM, JCAP 11 (2011) 027 [arXiv:1108.0978] [INSPIRE].

[45] D.G. Cerdeño, V. Martín-Lozano and O. Seto, Displaced vertices and long-lived charged particles in the NMSSM with right-handed sneutrinos, JHEP 05 (2014) 035 [arXiv:1311.7260] [INSPIRE].

[46] D.G. Cerdeño, M. Peiró and S. Robles, Low-mass right-handed sneutrino dark matter: SuperCDMS and LUX constraints and the galactic centre gamma-ray excess, JCAP 08 (2014) 005 [arXiv: 1404.2572] [INSPIRE].

[47] K.-Y. Choi, E.J. Chun and C.S. Shin, Dark matter asymmetry in supersymmetric Dirac leptogenesis, Phys. Lett. B 723 (2013) 90 [arXiv:1211.5409] [INSPIRE].

[48] Z. Kang, J. Li, T. Li, T. Liu and J. Yang, Asymmetric sneutrino dark matter in the NMSSM with minimal inverse seesaw, arXiv:1102.5644 [INSPIRE].

[49] J. Guo, Z. Kang, T. Li and Y. Liu, Higgs boson mass and complex sneutrino dark matter in the supersymmetric inverse seesaw models, JHEP 02 (2014) 080 [arXiv:1311.3497] [INSPIRE].

[50] S. Kanemura, N. Machida and T. Shindou, Radiative neutrino mass, dark matter and electroweak baryogenesis from the supersymmetric gauge theory with confinement, arXiv:1405.5834 [INSPIRE].

[51] LUX collaboration, D.S. Akerib et al., First results from the LUX dark matter experiment at the Sanford Underground Research Facility, Phys. Rev. Lett. 112 (2014) 091303 [arXiv: 1310.8214] [INSPIRE].

[52] S. Profumo and L. Ubaldi, Cosmic ray-dark matter scattering: a new signature of (asymmetric) dark matter in the gamma ray sky, JCAP 08 (2011) 020 [arXiv:1106.4568] [INSPIRE].

[53] A. Aboubrahim, T. Ibrahim and P. Nath, Radiative decays of cosmic background neutrinos in extensions of MSSM with a vector like lepton generation, Phys. Rev. D 88 (2013) 013019 [arXiv: 1306.2275] [INSPIRE].

[54] http://map.fnal.gov/.

[55] J. Hisano, T. Moroi, K. Tobe and M. Yamaguchi, Lepton flavor violation via right-handed neutrino Yukawa couplings in supersymmetric standard model, Phys. Rev. D 53 (1996) 2442 [hep-ph/9510309] [INSPIRE].

[56] J. Hisano and K. Tobe, Neutrino masses, muon $g-2$ and lepton flavor violation in the supersymmetric seesaw model, Phys. Lett. B 510 (2001) 197 [hep-ph/0102315] [INSPIRE]. 